\title{
Photo-induced Reactions of Diruthenium Tetrahydride Complexes: Carbon-Hydrogen Bond Cleavage of Tetrahydrofuran Leading to Bridging Cyclic Fischer-Type Carbene Complexes
}

Ryuichi Shimogawa ${ }^{\dagger}$, Gen-ichi Konishi ${ }^{\star}$, Toshiro Takao, ${ }^{\dagger,},{ }^{*}$ and Hiroharu Suzuki ${ }^{\dagger}$

${ }^{\dagger}$ Department of Applied Chemistry, Graduate School of Science and Engineering, Tokyo Institute of Technology,

2-12-1 O-okayama, Meguro-ku, Tokyo 152-8552, Japan

${ }^{\star}$ Department of Organic and Polymeric Materials, Graduate School of Science and Engineering, Tokyo Institute of Technology,

2-12-1 O-okayama, Meguro-ku, Tokyo 152-8552, Japan

${ }^{\S}$ JST, ACT-C, 4-1-8 Honcho, Kawaguchi, Saitama 3332-0012, Japans

\section{Supporting Information}

\section{Contents}

1. Crystal data and results of XRD studies $\mathbf{5 a}, \mathbf{5 b}, \mathbf{5 c}$, cis-8a, trans-8a, $9 \mathbf{a}$, and $9 \mathbf{c}$.

2. ORTEP diagram of $\mathbf{5 a}, \mathbf{5 b}, \mathbf{5} \mathbf{c}$, cis-8a, trans-8a, $9 \mathbf{a}, 9 \mathbf{c}$, and $\mathbf{1 0 a}$.

3. NMR simulation of cis-8a, trans-8a, 9a, and 9c.

4. VT-NMR spectra of $\mathbf{5 a}$ and $\mathbf{5 c}$.

5. NMR spectra of $\mathbf{5 a}, \mathbf{5 b}, \mathbf{5 c}$, cis-8a, trans-8a, $9 \mathbf{a}, \mathbf{9 c}$, and $\mathbf{1 0 a}$. 


\section{Crystal data and results of XRD studies.}

Table S1. Crystallographic data.

\begin{tabular}{|c|c|c|c|c|}
\hline & $5 a$ & $\mathbf{5 b}$ & $5 c$ & cis-8a \\
\hline Formula & $\mathrm{C}_{24} \mathrm{H}_{38} \mathrm{ORu}_{2}$ & $\mathrm{C}_{26} \mathrm{H}_{42} \mathrm{ORu}_{2}$ & $\mathrm{C}_{38} \mathrm{H}_{66} \mathrm{ORu}_{2}$ & $\mathrm{C}_{34} \mathrm{H}_{54} \mathrm{~N}_{2} \mathrm{ORu}_{2}$ \\
\hline Formula weight & 544.68 & 572.73 & 741.04 & 708.93 \\
\hline Crystal description & block & platelet & block & block \\
\hline Crystal color & brown & brown & brown & orange \\
\hline Crystal size (mm) & $0.170 \times 0.144 \times 0.142$ & $0.303 \times 0.224 \times 0.067$ & $0.321 \times 0.183 \times 0.173$ & $0.422 \times 0.347 \times 0.246$ \\
\hline Crystallizing solution & $\mathrm{THF}\left(-30^{\circ} \mathrm{C}\right)$ & pentane $\left(-30^{\circ} \mathrm{C}\right)$ & acetone $\left(-30^{\circ} \mathrm{C}\right)$ & pentane $\left(-30^{\circ} \mathrm{C}\right)$ \\
\hline Crystal system & triclinic & triclinic & monoclinic & triclinic \\
\hline Space group & $P-1(\# 2)$ & $P-1(\# 2)$ & $\operatorname{Pn}(\# 7)$ & $P-1(\# 2)$ \\
\hline$a(\AA)$ & $8.5051(3)$ & $8.5163(8)$ & $10.2806(6)$ & $11.273(2)$ \\
\hline$b(\AA)$ & $10.0519(4)$ & $11.2111(11)$ & $11.8219(7)$ & $12.396(2)$ \\
\hline$c(\AA)$ & $13.1631(5)$ & $13.2210(13)$ & $15.4868(10)$ & $12.556(2)$ \\
\hline$\alpha\left({ }^{\circ}\right)$ & $88.1100(10)$ & $88.343(2)$ & & $85.370(3)$ \\
\hline$\beta\left(^{\circ}\right)$ & $88.3640(10)$ & $87.845(3)$ & $103.059(2)$ & $80.494(4)$ \\
\hline$\gamma\left({ }^{\circ}\right)$ & $88.2110(10)$ & $85.305(3)$ & & $80.253(3)$ \\
\hline$V\left(\AA^{3}\right)$ & $1123.76(7)$ & $1256.8(2)$ & 1833.53(19) & $1702.8(5)$ \\
\hline$Z$ value & 2 & 2 & 2 & 2 \\
\hline$\rho_{\text {calcd }}\left(\mathrm{g} / \mathrm{cm}^{3}\right)$ & 1.610 & 1.513 & 1.342 & 1.383 \\
\hline $\begin{array}{l}\text { Measurement } \\
\text { temperature. }\left({ }^{\circ} \mathrm{C}\right)\end{array}$ & -150 & -150 & -150 & -150 \\
\hline$\mu($ Мок $\alpha)\left(\mathrm{mm}^{-1}\right)$ & 1.355 & 1.215 & 0.850 & 0.913 \\
\hline $2 \theta_{\max }(\mathrm{deg})$ & 55 & 55 & 55 & 55 \\
\hline $\begin{array}{l}\text { No. of reflections } \\
\text { collected }\end{array}$ & 9248 & 10372 & 17803 & 13969 \\
\hline $\begin{array}{l}\text { No. of unique } \\
\text { reflections }\end{array}$ & $4093\left(R_{\mathrm{int}}=0.0302\right)$ & $4600\left(R_{\text {int }}=0.0820\right)$ & $8224\left(R_{\mathrm{int}}=0.0320\right)$ & $6209\left(R_{\text {int }}=0.0520\right)$ \\
\hline $\begin{array}{l}\text { No. Reflections } \\
\text { observed }(>2 \sigma)\end{array}$ & 3539 & 3992 & 8063 & 5803 \\
\hline Abs. correction type & Empirical & Numerical & Numerical & Numerical \\
\hline Abs. transmission & $\begin{array}{l}0.6806 \text { (min.) } 1.0000 \\
\quad(\max .)\end{array}$ & $\begin{array}{l}0.7237 \text { (min.) } 0.8934 \\
\quad \text { (max.) }\end{array}$ & $\begin{array}{l}0.8124 \text { (min.) } 0.8787 \\
\quad(\max .)\end{array}$ & $\begin{array}{l}0.7543 \text { (min.) } 1.0000 \\
\quad(\max .)\end{array}$ \\
\hline$R_{1}[I>2 \sigma(I)]$ & 0.0269 & 0.0527 & 0.0275 & 0.0309 \\
\hline$w R_{2}[I>2 \sigma(I)]$ & 0.0619 & 0.1325 & 0.0703 & 0.0789 \\
\hline$R_{1}$ (all data) & 0.0339 & 0.0590 & 0.0283 & 0.0327 \\
\hline$w R_{2}$ (all data) & 0.0653 & 0.1387 & 0.0711 & 0.0803 \\
\hline $\begin{array}{l}\text { Data / restraints / } \\
\text { parameters }\end{array}$ & 4093 / 0 / 346 & $4600 / 0 / 344$ & 8224 / 2 / 465 & $6209 / 0 / 418$ \\
\hline Goodness of fit on $F^{2}$ & 1.051 & 1.053 & 1.044 & 1.026 \\
\hline $\begin{array}{l}\text { Largest diff. peak and } \\
\text { hole }\end{array}$ & 0.638 and $-0.450 \mathrm{e} \cdot \AA^{-3}$ & 0.890 and $-1.037 \mathrm{e} \cdot \AA^{-3}$ & 0.462 and $-0.470 \mathrm{e} \cdot \AA^{-3}$ & 0.435 and $-0.475 \mathrm{e} \cdot \AA^{-3}$ \\
\hline CCDC Number & 1450114 & 1450115 & 1450116 & 1450117 \\
\hline
\end{tabular}


Table S1. Crystallographic data. (continued)

\begin{tabular}{|c|c|c|c|}
\hline & trans-8a & $9 a$ & $9 c$ \\
\hline Formula & $\mathrm{C}_{34} \mathrm{H}_{54} \mathrm{~N}_{2} \mathrm{ORu}_{2}$ & $\mathrm{C}_{26} \mathrm{H}_{36} \mathrm{O}_{3} \mathrm{Ru}_{2}$ & $\mathrm{C}_{40} \mathrm{H}_{64} \mathrm{O}_{3} \mathrm{Ru}_{2}$ \\
\hline Formula weight & 708.93 & 598.69 & 795.05 \\
\hline Crystal description & block & platelet & platelet \\
\hline Crystal color & orange & yellow & orange \\
\hline Crystal size (mm) & $0.175 \times 0.096 \times 0.062$ & $0.477 \times 0.258 \times 0.087$ & $0.326 \times 0.221 \times 0.115$ \\
\hline Crystallizing solution & acetone $\left(-30^{\circ} \mathrm{C}\right)$ & hexane $\left(-30^{\circ} \mathrm{C}\right)$ & hexane $\left(-30^{\circ} \mathrm{C}\right)$ \\
\hline Crystal system & monoclinic & monoclinic & monoclinic \\
\hline Space group & $P 2{ }_{1} / n(\# 14)$ & $P 2{ }_{1} / n(\# 14)$ & $P 2{ }_{1} / c$ (\#14) \\
\hline$a(\AA)$ & $13.2186(4)$ & $8.5953(3)$ & $19.5372(5)$ \\
\hline$b(\AA)$ & $17.8772(5)$ & $20.2385(6)$ & $9.2054(3)$ \\
\hline$c(\AA)$ & $14.3598(4)$ & $14.6209(6)$ & $23.5257(6)$ \\
\hline$\alpha\left({ }^{\circ}\right)$ & & & \\
\hline$\beta\left(^{\circ}\right)$ & $97.2790(10)$ & $99.5490(10)$ & $111.3880(10)$ \\
\hline$\gamma\left({ }^{\circ}\right)$ & & & \\
\hline$V\left(\AA^{3}\right)$ & $3366.04(17)$ & $2508.15(15)$ & $3939.66(19)$ \\
\hline$Z$ value & 4 & 4 & 4 \\
\hline$\rho_{\text {calcd }}\left(\mathrm{g} / \mathrm{cm}^{3}\right)$ & 1.399 & 1.585 & 1.340 \\
\hline $\begin{array}{l}\text { Measurement } \\
\text { temperature. }\left({ }^{\circ} \mathrm{C}\right)\end{array}$ & -150 & -130 & -150 \\
\hline$\mu(\mathrm{MoK} \alpha)\left(\mathrm{mm}^{-1}\right)$ & 0.924 & 1.228 & 0.800 \\
\hline $2 \theta_{\max }(\mathrm{deg})$ & 55 & 55 & 55 \\
\hline $\begin{array}{l}\text { No. of reflections } \\
\text { collected }\end{array}$ & 53196 & 24455 & 61273 \\
\hline $\begin{array}{l}\text { No. of unique } \\
\text { reflections }\end{array}$ & $7690\left(R_{\mathrm{int}}=0.0378\right)$ & $5734\left(R_{\mathrm{int}}=0.0366\right)$ & $8964\left(R_{\mathrm{int}}=0.0517\right)$ \\
\hline $\begin{array}{l}\text { No. Reflections } \\
\text { observed }(>2 \sigma)\end{array}$ & $6880 \mathrm{~s}$ & 5222 & 7459 \\
\hline Abs. correction type & Empirical & Numerical & Numerical \\
\hline Abs. transmission & $\begin{array}{l}0.7142 \text { (min.) } 1.0000 \\
\quad \text { (max.) }\end{array}$ & $\begin{array}{l}0.6498 \text { (min.) } 0.8717 \\
\quad(\max .)\end{array}$ & $\begin{array}{l}0.8357 \text { (min.) } 0.9238 \\
\quad(\max .)\end{array}$ \\
\hline$R_{1}[I>2 \sigma(I)]$ & 0.0242 & 0.0203 & 0.0256 \\
\hline$w R_{2}[I>2 \sigma(I)]$ & 0.0590 & 0.0456 & 0.0547 \\
\hline$R_{1}$ (all data) & 0.0294 & 0.0238 & 0.0352 \\
\hline$w R_{2}$ (all data) & 0.0616 & 0.0469 & 0.0576 \\
\hline $\begin{array}{l}\text { Data / restraints / } \\
\text { parameters }\end{array}$ & $7690 / 0 / 392$ & $5734 / 0 / 298$ & 8964 / 0 / 448 \\
\hline Goodness of fit on $F^{2}$ & 1.013 & 1.052 & 1.043 \\
\hline $\begin{array}{l}\text { Largest diff. peak and } \\
\text { hole }\end{array}$ & 1.076 and $-0.404 \mathrm{e} \cdot \AA^{-3}$ & 0.404 and $-0.342 \mathrm{e} \cdot \AA^{-3}$ & 0.408 and $-0.640 \mathrm{e} \cdot \AA^{-3}$ \\
\hline CCDC Number & 1450118 & 1450119 & 1450120 \\
\hline
\end{tabular}




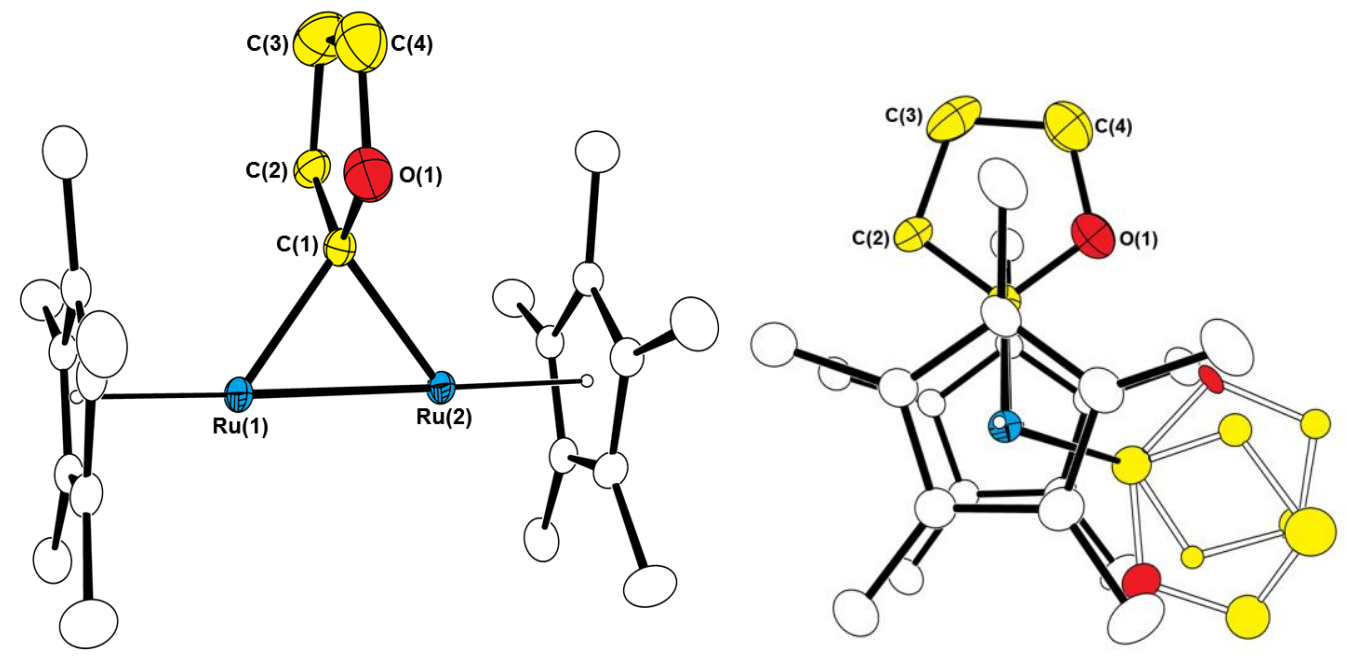

Figure S1. Molecular Structure of $\mathbf{5 a}$ with thermal ellipsoids set at $30 \%$ probability. Hydrogen atoms are omitted for clarity. The two disordered carbene ligands with smaller occupancies are omitted for clarity (left top). 5a has disordered structures (occupancies; 50, 25, and 25\%) with respect to the orientation of the carbene ligand (right). Selected bond lengths (A) and angles $\left(^{\circ}\right)$ : $\mathrm{Ru}(1)-\mathrm{Ru}(2), 2.4167(3) ; \mathrm{Ru}(1)-\mathrm{C}(1), 2.048(6) ; \mathrm{Ru}(2)-\mathrm{C}(1), 2.048(6) ; \mathrm{O}(1)-\mathrm{C}(1), 1.440(8) ; \mathrm{C}(1)-\mathrm{C}(2), 1.474(8)$; $\mathrm{C}(2)-\mathrm{C}(3), 1.590(11) ; \mathrm{C}(3)-\mathrm{C}(4), 1.492(13) ; \mathrm{C}(4)-\mathrm{O}(1), 1.467(9) ; \mathrm{C}(1)-\mathrm{Ru}(1)-\mathrm{Ru}(2), 53.83(17) ; \mathrm{C}(1)-\mathrm{Ru}(2)-\mathrm{Ru}(1)$, 53.84(18); $\mathrm{Ru}(1)-\mathrm{C}(1)-\mathrm{Ru}(2), 72.3(2) ; \mathrm{C}(4)-\mathrm{O}(1)-\mathrm{C}(1), 113.1(5) ; \mathrm{O}(1)-\mathrm{C}(1)-\mathrm{C}(2), 107.5(5) ; \mathrm{C}(1)-\mathrm{C}(2)-\mathrm{C}(3), 106.8(5)$; $\mathrm{C}(2)-\mathrm{C}(3)-\mathrm{O}(4), 105.9(6) ; \mathrm{C}(3)-\mathrm{C}(4)-\mathrm{O}(1), 106.6(6)$.
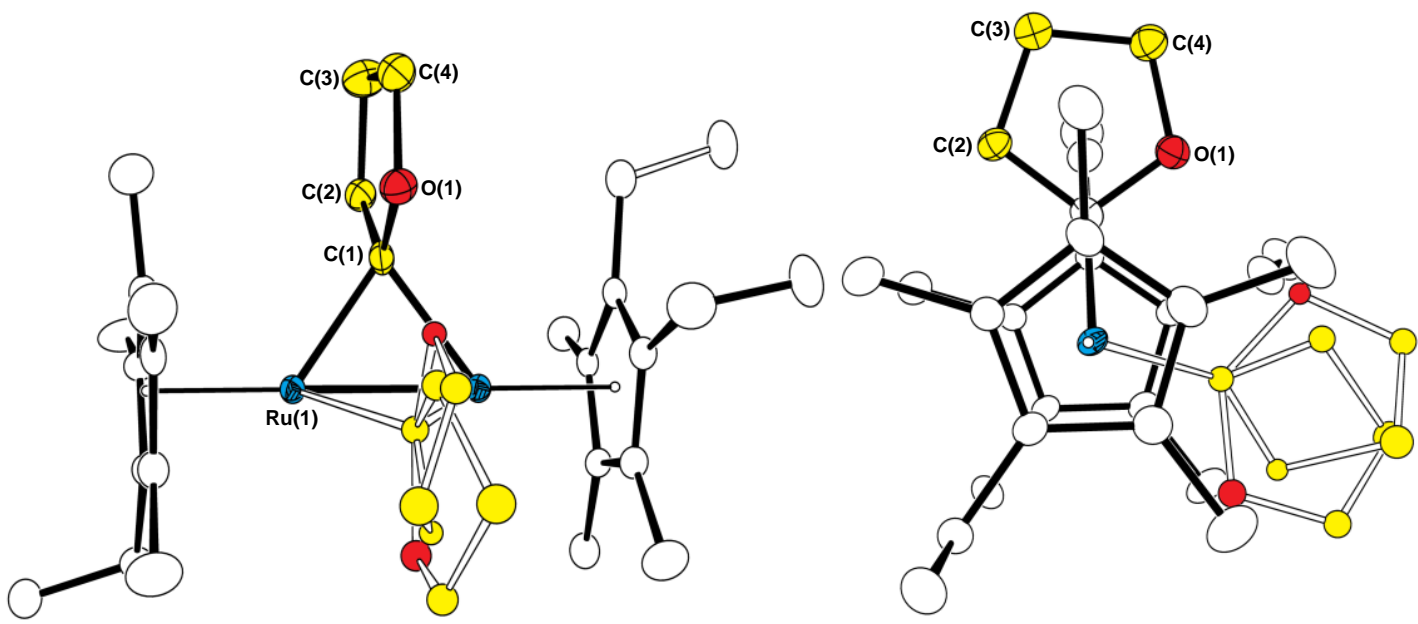

Figure S2. Molecular Structure of $\mathbf{5 b}$ with thermal ellipsoids set at $30 \%$ probability. Hydrogen atoms are omitted for clarity. $\mathbf{5 b}$ has disordered structures with respect to the orientation of the carbene ligand (occupancies; 46, 27, and 27\%) and ethyl group (occupancies; 48 and 52\%). Selected bond lengths $(\AA)$ and angles $\left({ }^{\circ}\right)$ : $\operatorname{Ru}(1)-\operatorname{Ru}(2), 2.4120(5) ; \operatorname{Ru}(1)-C(1), 2.042(10)$; $\mathrm{Ru}(2)-\mathrm{C}(1), 2.081(10) ; \mathrm{O}(1)-\mathrm{C}(1), 1.377(11) ; \mathrm{C}(1)-\mathrm{C}(2), 1.502(14) ; \mathrm{C}(2)-\mathrm{C}(3), 1.558(15) ; \mathrm{C}(3)-\mathrm{C}(4), 1.507(17) ; \mathrm{C}(4)-\mathrm{O}(1)$, 1.469(13); C(1)-Ru(1)-Ru(2), 55.0(3); C(1)-Ru(2)-Ru(1), 53.4(3); Ru(1)-C(1)-Ru(2), 71.6(3); C(4)-O(1)-C(1), 113.3(8); $\mathrm{O}(1)-\mathrm{C}(1)-\mathrm{C}(2), 107.3(8) ; \mathrm{C}(1)-\mathrm{C}(2)-\mathrm{C}(3), 108.0(9) ; \mathrm{C}(2)-\mathrm{C}(3)-\mathrm{O}(4), 103.6(9) ; \mathrm{C}(3)-\mathrm{C}(4)-\mathrm{O}(1), 107.4(9)$. 

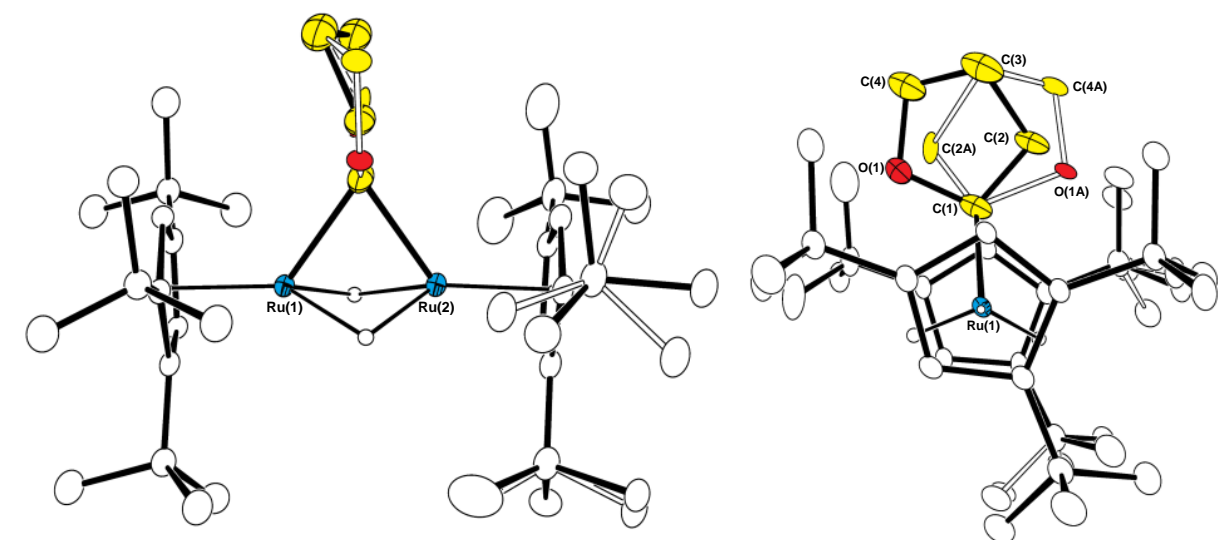

Figure S3. Molecular Structure of 5c with thermal ellipsoids set at $30 \%$ probability. Hydrogen atoms besides the hydrides are omitted for clarity. $\mathbf{5 c}$ has disordered structures with respect to the orientation of the carbene ligand (occupancies; 78 and $22 \%$ ) and two $t$-butyl groups (occupancies; 57 and 43\%, 61 and 39\%). Selected bond lengths $(\AA)$ and angles $\left({ }^{\circ}\right): \operatorname{Ru}(1)-\operatorname{Ru}(2)$, 2.4107(4); Ru(1)-C(1), 2.117(5); Ru(2)-C(1), 2.095(5); O(1)-C(1), 1.464(9); C(1)-C(2), 1.473(9); C(2)-C(3), 1.596(15); C(3)$\mathrm{C}(4), 1.429(13) ; \mathrm{C}(4)-\mathrm{O}(1), 1.458(9) ; \mathrm{C}(1)-\mathrm{Ru}(1)-\mathrm{Ru}(2), 54.66(14) ; \mathrm{C}(1)-\mathrm{Ru}(2)-\mathrm{Ru}(1), 55.51(13) ; \mathrm{Ru}(1)-\mathrm{C}(1)-\mathrm{Ru}(2)$, 69.82(15); C(4)-O(1)-C(1), 109.4(7); O(1)-C(1)-C(2), 106.1(7); C(1)-C(2)-C(3), 102.7(8); C(2)-C(3)-O(4), 102.3(6); C(3)$\mathrm{C}(4)-\mathrm{O}(1), 105.7(6)$.

$\mathrm{S}$

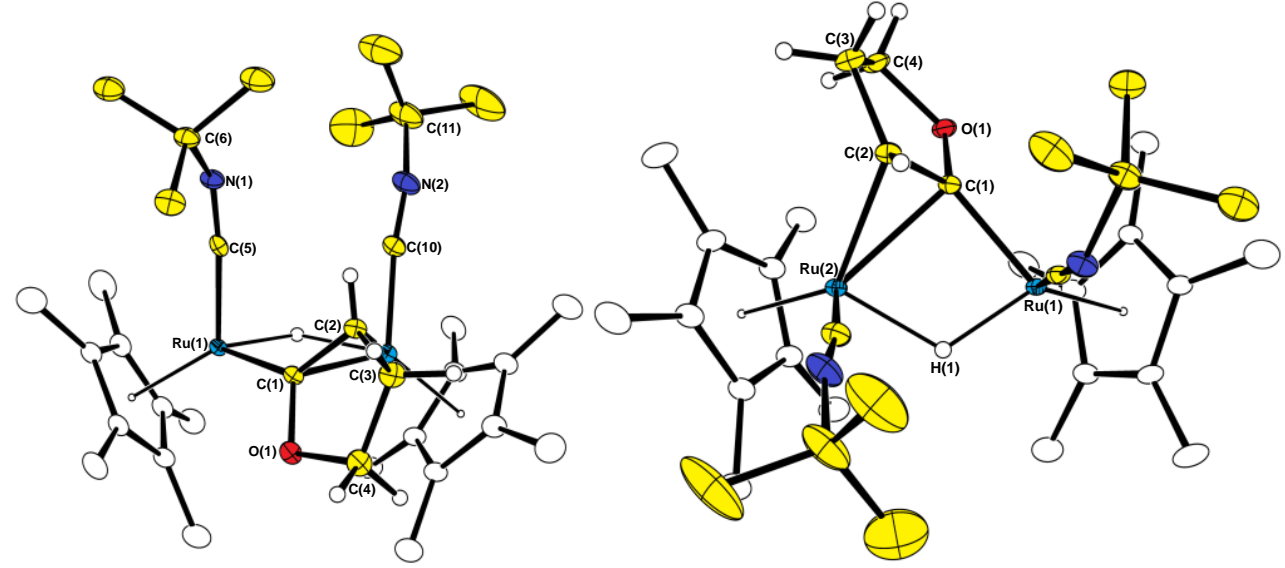

Figure S4. Molecular Structure of cis-8a with thermal ellipsoids set at $30 \%$ probability. Hydrogen atoms of the Cp* and

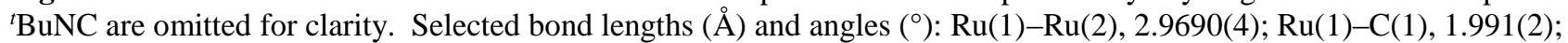
$\mathrm{Ru}(2)-\mathrm{C}(1), 2.270(2) ; \mathrm{Ru}(2)-\mathrm{C}(2), 2.248(2) ; \mathrm{O}(1)-\mathrm{C}(1), 1.398(3) ; \mathrm{C}(1)-\mathrm{C}(2), 1.409(3) ; \mathrm{C}(2)-\mathrm{C}(3), 1.513(3) ; \mathrm{C}(3)-\mathrm{C}(4)$, 1.507(4); C(4)-O(1), 1.448(3); Ru(1)-C(5), 1.863(3); N(1)-C(5), 1.192(3); N(1)-C(6), 1.479(3); Ru(2)-C(10), 1.897(3); $\mathrm{N}(2)-\mathrm{C}(10), 1.164(3) ; \mathrm{N}(2)-\mathrm{C}(11), 1.434(4) ; \mathrm{Ru}(1)-\mathrm{C}(1)-\mathrm{Ru}(2), 88.10(8) ; \mathrm{O}(1)-\mathrm{C}(1)-\mathrm{Ru}(1), 120.15(16) ; \mathrm{Ru}(1)-\mathrm{C}(1)-\mathrm{C}(2)$, 130.57(18); C(4)-O(1)-C(1), 109.41(18); O(1)-C(1)-C(2), 109.2(2); C(1)-C(2)-C(3), 109.1(2); C(2)-C(3)-O(4), 101.9(2); $\mathrm{C}(3)-\mathrm{C}(4)-\mathrm{O}(1), 106.9(2) ; \mathrm{Ru}(1)-\mathrm{C}(5)-\mathrm{N}(1), 174.1(2) ; \mathrm{C}(5)-\mathrm{N}(1)-\mathrm{C}(6), 134.4(2) ; \mathrm{Ru}(2)-\mathrm{C}(10)-\mathrm{N}(2), 172.0(2) ; \mathrm{C}(10)-\mathrm{N}(2)-$ $\mathrm{C}(11), 160.2(3)$. 

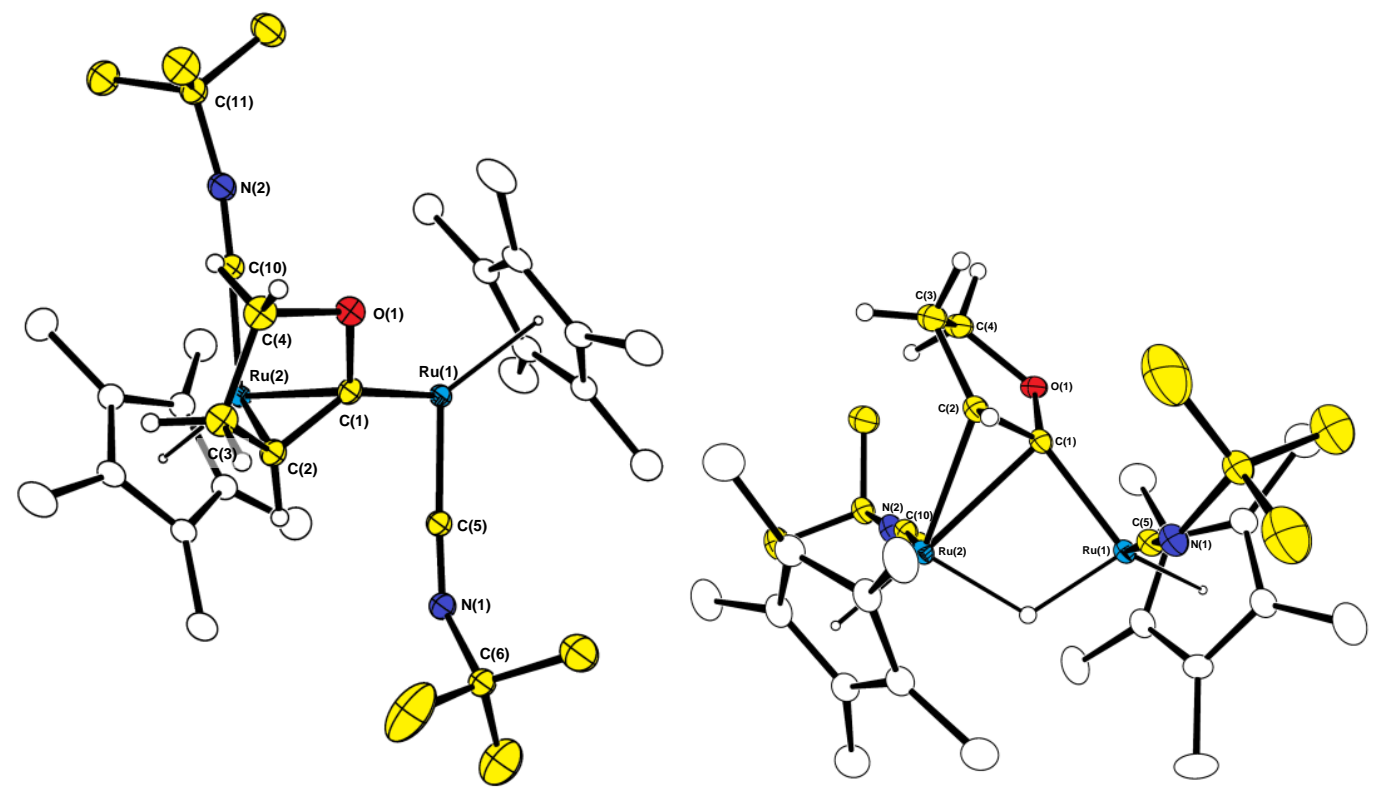

Figure S5. Molecular Structure of trans-8a with thermal ellipsoids set at $30 \%$ probability. Hydrogen atoms of the Cp* and ${ }^{t} \mathrm{BuNC}$ are omitted for clarity. Selected bond lengths $(\AA)$ and angles $\left({ }^{\circ}\right)$ : $\mathrm{Ru}(1)-\mathrm{Ru}(2), 2.9245(2) ; \mathrm{Ru}(1)-\mathrm{C}(1), 2.0096(19)$; $\mathrm{Ru}(2)-\mathrm{C}(1), 2.3465(19) ; \mathrm{Ru}(2)-\mathrm{C}(2), 2.2499(19) ; \mathrm{O}(1)-\mathrm{C}(1), 1.393(2) ; \mathrm{C}(1)-\mathrm{C}(2), 1.402(3) ; \mathrm{C}(2)-\mathrm{C}(3), 1.509(3) ; \mathrm{C}(3)-\mathrm{C}(4)$, 1.525(3); C(4)-O(1), 1.452(2); Ru(1)-C(5), 1.860(2); N(1)-C(5), 1.200(3); N(1)-C(6), 1.473(3); Ru(2)-C(10), 1.910(2); $\mathrm{N}(2)-\mathrm{C}(10), 1.171(3) ; \mathrm{N}(2)-\mathrm{C}(11), 1.454(2) ; \mathrm{Ru}(1)-\mathrm{C}(1)-\mathrm{Ru}(2), 83.96(7) ; \mathrm{O}(1)-\mathrm{C}(1)-\mathrm{Ru}(1), 119.67(13) ; \mathrm{Ru}(1)-\mathrm{C}(1)-\mathrm{C}(2)$, 130.64(15); C(4)-O(1)-C(1), 109.09(15); O(1)-C(1)-C(2), 109.52(16); C(1)-C(2)-C(3), 109.50(18); C(2)-C(3)-O(4), 101.12(17); C(3)-C(4)-O(1), 106.15(16); Ru(1)-C(5)-N(1), 176.19(18); C(5)-N(1)-C(6), 135.91(19); Ru(2)-C(10)-N(2), 171.25(18); C(10)-N(2)-C(11), 165.9(2).
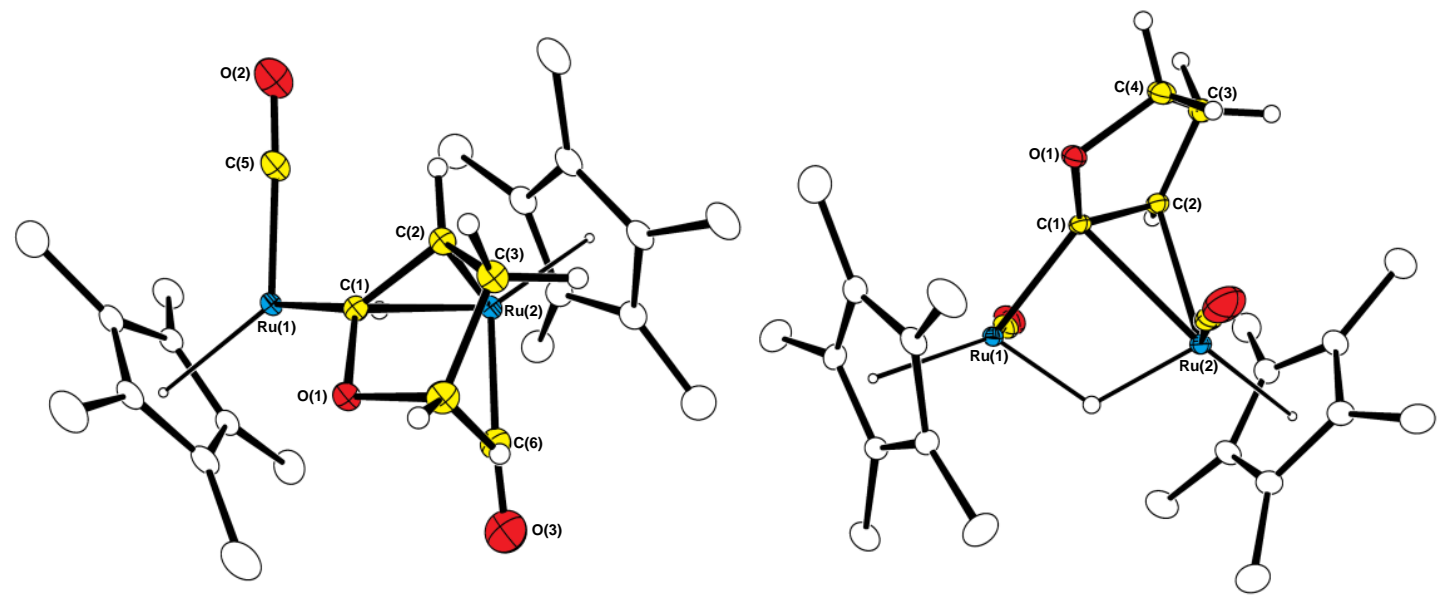

Figure S6. Molecular Structure of 9a with thermal ellipsoids set at $30 \%$ probability. Hydrogen atoms of the Cp* are omitted for clarity. Selected bond lengths $(\AA)$ and angles $\left(^{\circ}\right): \operatorname{Ru}(1)-\operatorname{Ru}(2), 2.91596(19) ; \operatorname{Ru}(1)-C(1), 1.9978(17) ; \mathrm{Ru}(2)-\mathrm{C}(1)$, 2.3821(17); Ru(2)-C(2), 2.2338(18); O(1)-C(1), 1.386(2); C(1)-C(2), 1.406(2); C(2)-C(3), 1.514(2); C(3)-C(4), 1.526(3); $\mathrm{C}(4)-\mathrm{O}(1), 1.456(2) ; \mathrm{Ru}(1)-\mathrm{C}(5), 1.8400(19) ; \mathrm{O}(2)-\mathrm{C}(5), 1.157(2) ; \mathrm{Ru}(2)-\mathrm{C}(6), 1.840(2) ; \mathrm{O}(3)-\mathrm{C}(6), 1.156(2) ; \mathrm{Ru}(1)-\mathrm{C}(1)-$ $\mathrm{Ru}(2), 82.98(6) ; \mathrm{O}(1)-\mathrm{C}(1)-\mathrm{Ru}(1), 120.47(11) ; \mathrm{Ru}(1)-\mathrm{C}(1)-\mathrm{C}(2), 129.36(13) ; \mathrm{C}(4)-\mathrm{O}(1)-\mathrm{C}(1), 109.52(13) ; \mathrm{O}(1)-\mathrm{C}(1)-\mathrm{C}(2)$, 109.71(14); C(1)-C(2)-C(3), 109.18(15); C(2)-C(3)-O(4), 101.39(14); C(3)-C(4)-O(1), 105.94(14); Ru(1)-C(5)-O(2), 177.22(18); $\mathrm{Ru}(2)-\mathrm{C}(6)-\mathrm{O}(3), 174.21(17)$. 

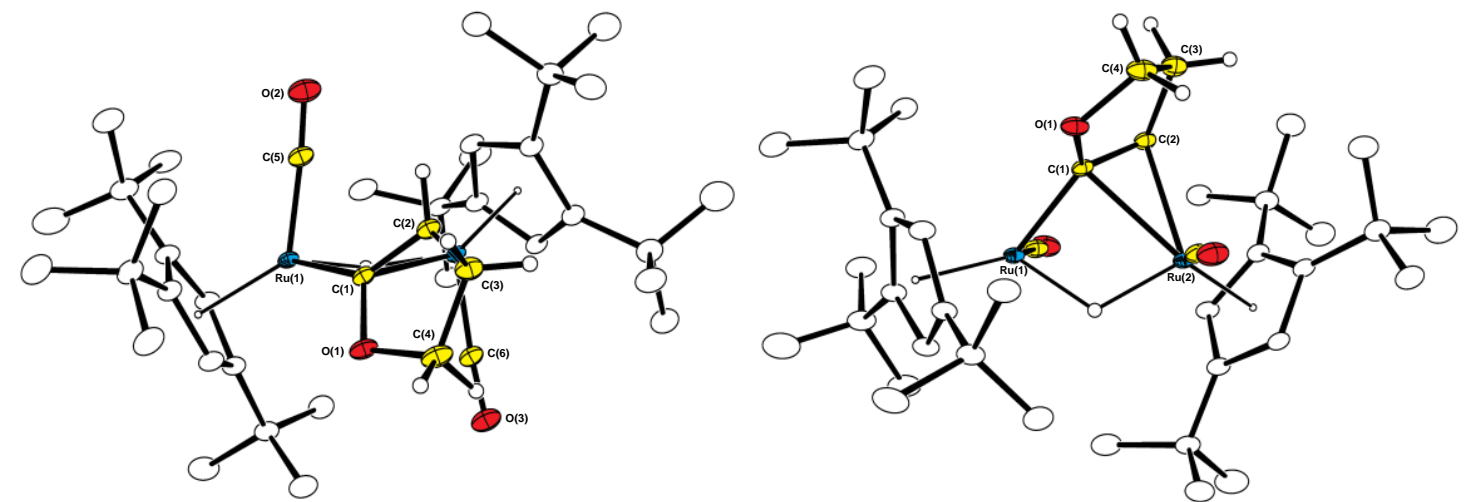

Figure S7. Molecular Structure of $\mathbf{9 c}$ with thermal ellipsoids set at $30 \%$ probability. Hydrogen atoms of the $\mathrm{Cp}^{\ddagger}$ are omitted for clarity. Selected bond lengths $(\AA)$ and angles $\left(^{\circ}\right): \mathrm{Ru}(1)-\mathrm{Ru}(2), 2.9740(2) ; \mathrm{Ru}(1)-\mathrm{C}(1), 1.9831(19) ; \mathrm{Ru}(2)-\mathrm{C}(1)$, 2.4075(18); $\mathrm{Ru}(2)-\mathrm{C}(2), 2.2607(18) ; \mathrm{O}(1)-\mathrm{C}(1), 1.382(2) ; \mathrm{C}(1)-\mathrm{C}(2), 1.405(3) ; \mathrm{C}(2)-\mathrm{C}(3), 1.524(3) ; \mathrm{C}(3)-\mathrm{C}(4), 1.513(3)$; $\mathrm{C}(4)-\mathrm{O}(1), 1.458(2) ; \mathrm{Ru}(1)-\mathrm{C}(5), 1.835(2) ; \mathrm{O}(2)-\mathrm{C}(5), 1.160(2) ; \mathrm{Ru}(2)-\mathrm{C}(6), 1.841(2) ; \mathrm{O}(3)-\mathrm{C}(6), 1.157(2) ; \mathrm{Ru}(1)-\mathrm{C}(1)-$ $\mathrm{Ru}(2), 84.69(6) ; \mathrm{O}(1)-\mathrm{C}(1)-\mathrm{Ru}(1), 118.43(13) ; \mathrm{Ru}(1)-\mathrm{C}(1)-\mathrm{C}(2), 131.17(14) ; \mathrm{C}(4)-\mathrm{O}(1)-\mathrm{C}(1), 109.43(15) ; \mathrm{O}(1)-\mathrm{C}(1)-\mathrm{C}(2)$, 110.12(16); $\mathrm{C}(1)-\mathrm{C}(2)-\mathrm{C}(3), 108.00(17) ; \mathrm{C}(2)-\mathrm{C}(3)-\mathrm{O}(4), 101.54(16) ; \mathrm{C}(3)-\mathrm{C}(4)-\mathrm{O}(1), 105.79(16) ; \mathrm{Ru}(1)-\mathrm{C}(5)-\mathrm{O}(2)$, 176.08(17); $\mathrm{Ru}(2)-\mathrm{C}(6)-\mathrm{O}(3), 173.56(16)$.

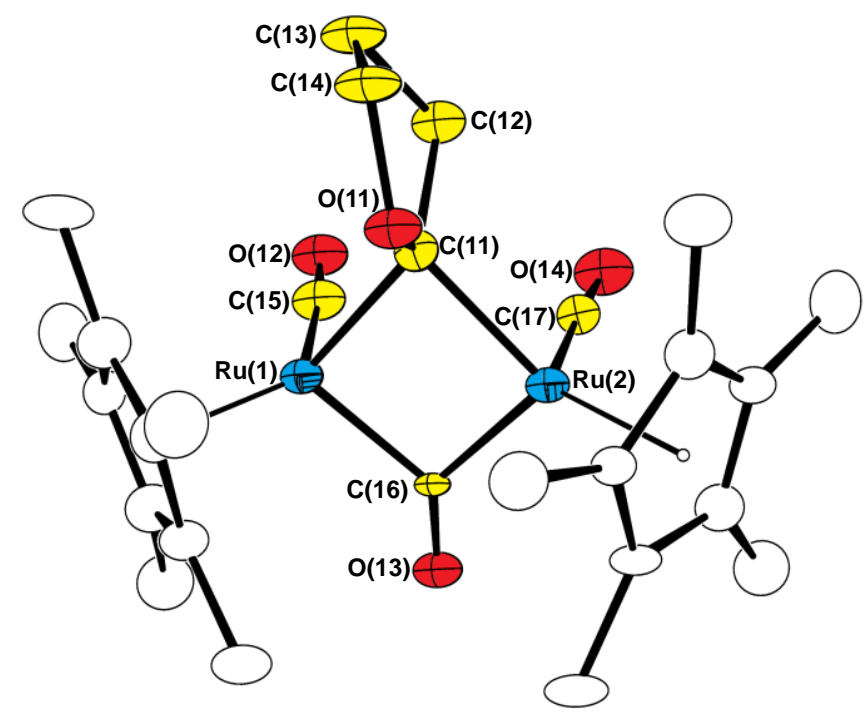

Figure S8. Molecular structure of 10a (preliminary) with thermal ellipsoids set at $30 \%$ probability. Hydrogen atoms are omitted for clarity.

$R_{1}=0.0958, w R_{2}=0.2263, P-1(\# 2)$, a: 8.1547(6), b: 23.3704(18), c: 26.9636(19), $\alpha: 87.880(2), \beta: 89.914(2), \gamma .89 .444(3)$. 
3 NMR simulation of cis-8a, trans-8a, 9a, and 9c.

(1) $c i s-8 \mathbf{a}$

Torsion angle of cis-8a determined from XRD study.

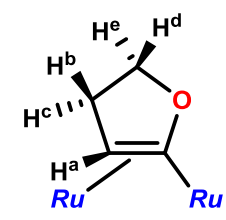

$\mathrm{H}^{\mathrm{a}}-\mathrm{C}-\mathrm{C}-\mathrm{H}^{\mathrm{c}}: 83.53$

$\mathrm{H}^{\mathrm{c}}-\mathrm{C}-\mathrm{C}-\mathrm{H}^{\mathrm{d}}: 102.47$

Table S2. Parameters for the simulation of ${ }^{1} \mathrm{H}$ NMR spectrum of $c$ is-8a.

\begin{tabular}{|l|c|c|c|c|c|c|} 
& $\boldsymbol{\delta}$ & $\boldsymbol{W}$ & \multicolumn{4}{c|}{$\boldsymbol{J}_{\mathbf{H H}}(\mathbf{H z})$} \\
\hline & $(\mathrm{ppm})$ & $(\mathrm{Hz})$ & $\mathrm{H}^{\mathrm{d}}$ & $\mathrm{H}^{\mathrm{e}}$ & $\mathrm{H}^{\mathrm{a}}$ & $\mathrm{H}^{\mathrm{b}}$ \\
\hline $\mathbf{H}^{\mathrm{d}}$ & 4.457 & 1.20 & & & & \\
\hline $\mathbf{H}^{\mathrm{e}}$ & 3.843 & 1.20 & -8.43 & & & \\
\hline $\mathbf{H}^{\mathrm{a}}$ & 3.003 & 1.20 & 0.00 & 0.00 & & \\
\hline $\mathbf{H}^{\mathbf{b}}$ & 2.566 & 1.20 & 10.70 & 8.45 & 5.65 & \\
\hline $\mathbf{H}^{\mathbf{c}}$ & 2.141 & 1.60 & 5.02 & 9.51 & 0.02 & -13.00 \\
\hline
\end{tabular}
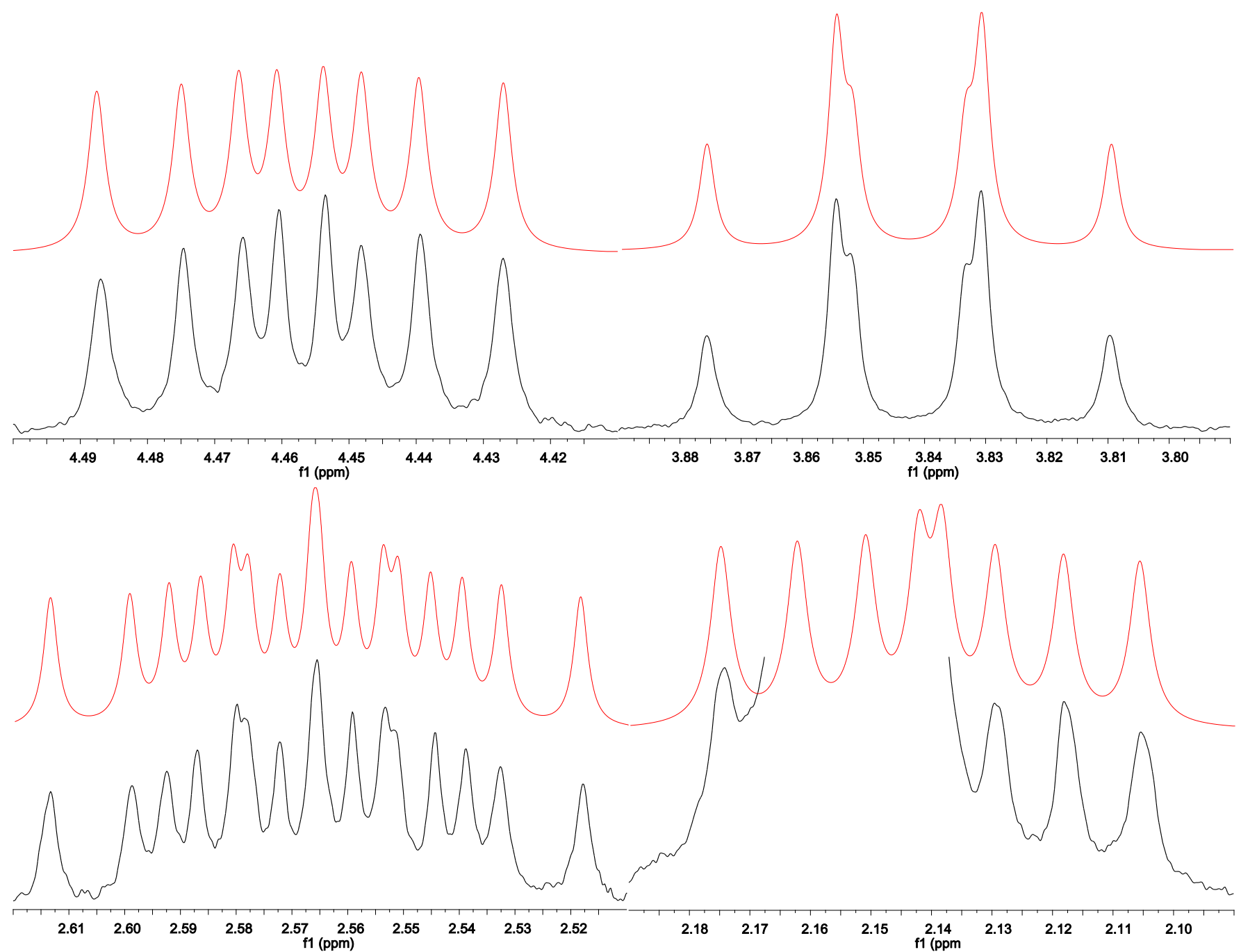

Figure S9. ${ }^{1} \mathrm{H}$ NMR sectra of cis-8a measured at $25{ }^{\circ} \mathrm{C}$ (Black) and results of the simulations (red) showing the dihydrofuranyl moiety region $\left(400 \mathrm{MHz},\left[\mathrm{D}_{6}\right.\right.$ ] benzene). The signal derived from $\mathrm{H}^{\mathrm{c}}$ is partly obscured by the strong $\mathrm{Cp}^{*}$ signal resonating at $\delta$ $2.16 \mathrm{ppm}$. 
(2) $\operatorname{trans}-\mathbf{8 a}$

Torsion angle of trans-8a determined from XRD study.

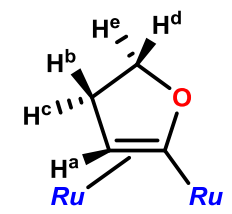

$\mathrm{H}^{\mathrm{a}}-\mathrm{C}-\mathrm{C}-\mathrm{H}^{\mathrm{c}}$ : 78.71

$\mathrm{H}^{\mathrm{c}}-\mathrm{C}-\mathrm{C}-\mathrm{H}^{\mathrm{d}}: 98.57$

Table S3. Parameters for the simulation of ${ }^{1} \mathrm{H}$ NMR spectrum of trans-8a.

\begin{tabular}{|c|c|c|c|c|c|c|} 
& $\boldsymbol{\delta}$ & $\boldsymbol{W}$ & \multicolumn{4}{c}{$\boldsymbol{J}_{\mathbf{H H}}(\mathbf{H z})$} \\
\hline & $(\mathrm{ppm})$ & $(\mathrm{Hz})$ & $\mathrm{H}^{\mathrm{d}}$ & $\mathrm{H}^{\mathrm{e}}$ & $\mathrm{H}^{\mathrm{b}}$ & $\mathrm{H}^{\mathrm{a}}$ \\
\hline $\mathbf{H}^{\mathbf{d}}$ & 4.479 & 1.40 & & & & \\
\hline $\mathbf{H}^{\mathrm{e}}$ & 4.105 & 1.40 & -7.50 & & & \\
\hline $\mathbf{H}^{\mathbf{b}}$ & 2.707 & 1.40 & 10.20 & 10.40 & & \\
\hline $\mathbf{H}^{\mathrm{a}}$ & 2.683 & 1.10 & -0.30 & 0.00 & 4.56 & \\
\hline $\mathbf{H}^{\mathbf{c}}$ & 2.087 & 1.40 & 2.80 & 9.10 & -12.12 & 0.00
\end{tabular}
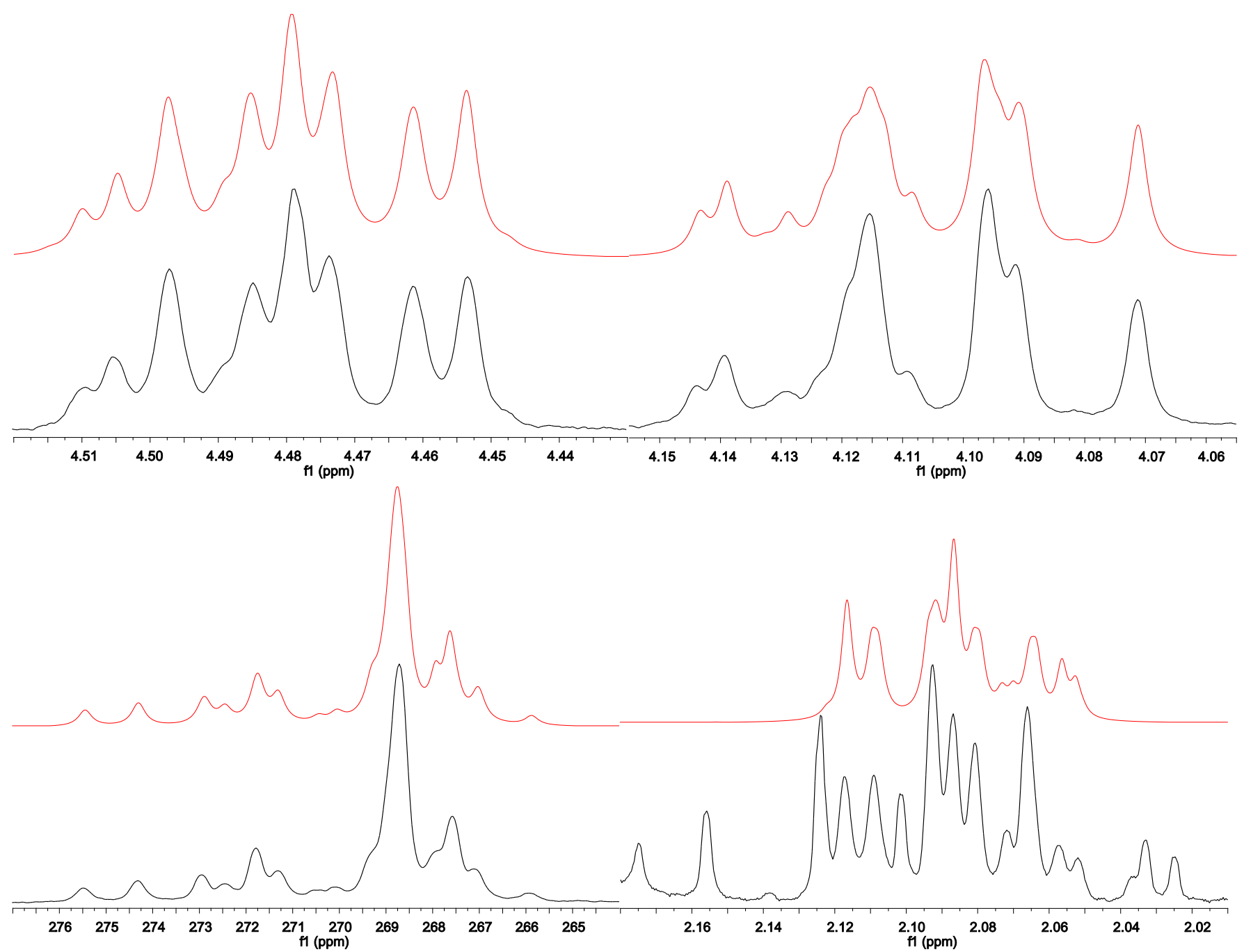

Figure S10. ${ }^{1} \mathrm{H}$ NMR sectra of trans-8a measured at $25^{\circ} \mathrm{C}$ (Black) and results of the simulations (red) showing the dihydrofuranyl moiety region $\left(400 \mathrm{MHz},\left[\mathrm{D}_{6}\right]\right.$ benzene). The signal derived from $\mathrm{H}^{\mathrm{c}}$ is partly obscured by the satellite peaks of $\mathrm{Cp} *$ signals and a small amount of impurities. 
(3) $9 \mathbf{a}$

Torsion angle of 9a determined from XRD study.

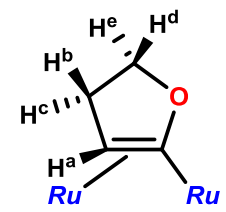

$\mathrm{H}^{\mathrm{a}}-\mathrm{C}-\mathrm{C}-\mathrm{H}^{\mathrm{c}}: 82.85$

$\mathrm{H}^{\mathrm{c}}-\mathrm{C}-\mathrm{C}-\mathrm{H}^{\mathrm{d}}: 101.23$

Table S4. Parameters for the simulation of ${ }^{1} \mathrm{H}$ NMR spectrum of $\mathbf{9 a}$.

\begin{tabular}{|c|c|c|c|c|c|c|} 
& $\boldsymbol{\delta}$ & $\boldsymbol{W}$ & \multicolumn{4}{c|}{$\boldsymbol{J H H}_{\mathbf{H}}(\mathbf{H z})$} \\
\hline & $(\mathrm{ppm})$ & $(\mathrm{Hz})$ & $\mathrm{H}^{\mathrm{d}}$ & $\mathrm{H}^{\mathrm{e}}$ & $\mathrm{H}^{\mathrm{a}}$ & $\mathrm{H}^{\mathrm{b}}$ \\
\hline $\mathbf{H}^{\mathbf{d}}$ & 4.442 & 0.80 & & & & \\
\hline $\mathbf{H}^{\mathrm{e}}$ & 4.165 & 1.00 & -8.50 & & & \\
\hline $\mathbf{H}^{\mathrm{a}}$ & 2.552 & 1.00 & 0.00 & 0.00 & & \\
\hline $\mathbf{H}^{\mathbf{b}}$ & 2.363 & 1.00 & 10.28 & 10.60 & 4.56 & \\
\hline $\mathbf{H}^{\mathrm{c}}$ & 1.844 & 1.10 & 2.70 & 9.16 & 0.00 & -12.80 \\
\hline
\end{tabular}
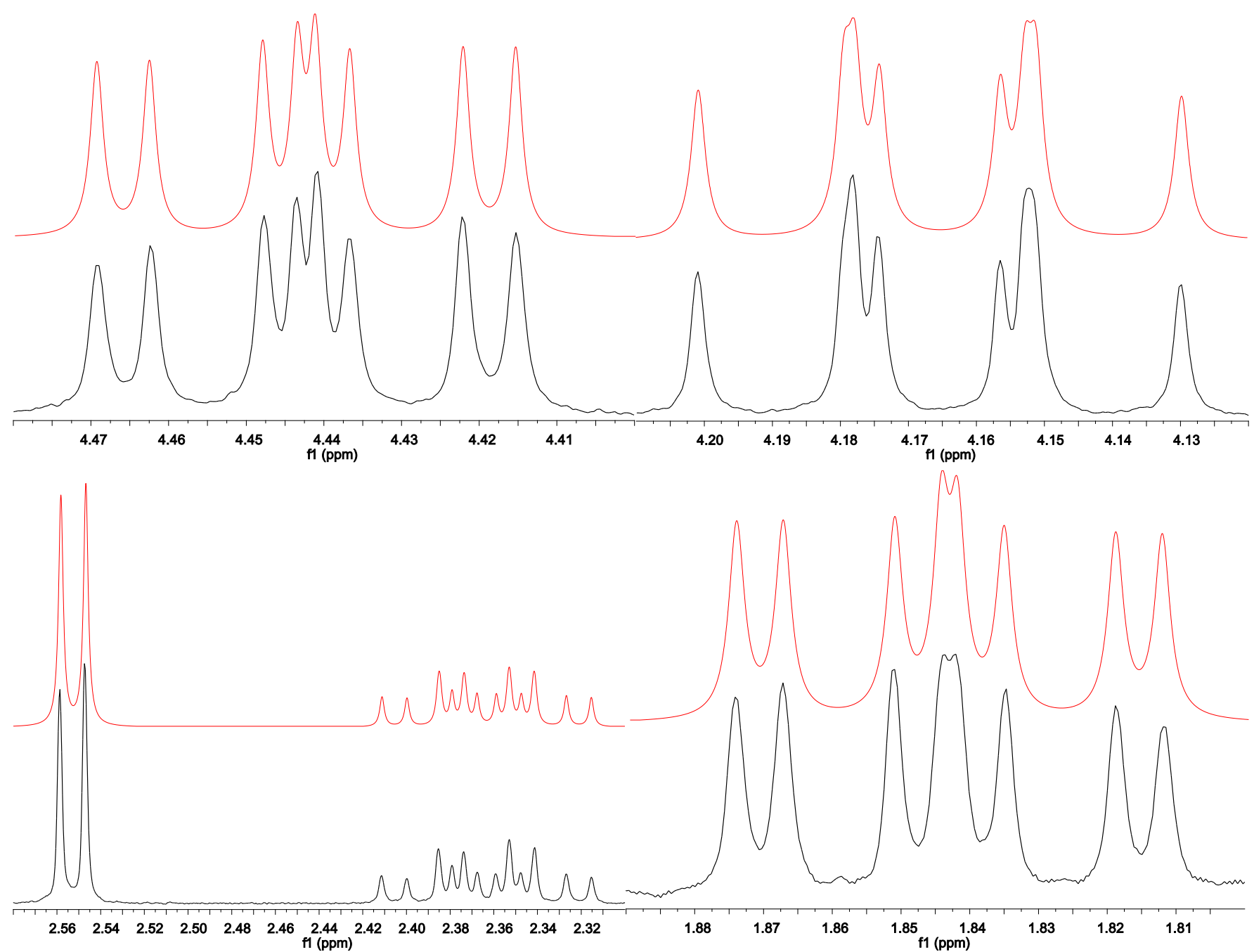

Figure S11. ${ }^{1} \mathrm{H}$ NMR sectra of 9a measured at $25^{\circ} \mathrm{C}$ (Black) and results of the simulations (red) showing the dihydrofuranyl moiety region $\left(400 \mathrm{MHz},\left[\mathrm{D}_{6}\right]\right.$ benzene). 
(4) $9 \mathrm{c}$

Torsion angle of trans-9c determined from XRD study.

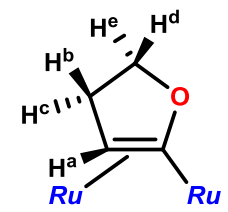

$\mathrm{H}^{\mathrm{a}}-\mathrm{C}-\mathrm{C}-\mathrm{H}^{\mathrm{c}}: 77.50$

$\mathrm{H}^{\mathrm{c}}-\mathrm{C}-\mathrm{C}-\mathrm{H}^{\mathrm{d}}: 97.14$

Table S5. Parameters for the simulation of ${ }^{1} \mathrm{H}$ NMR spectrum of $\mathbf{9 c}$.

\begin{tabular}{|c|c|c|c|c|c|c|} 
& \multicolumn{1}{c}{$\boldsymbol{\delta}$} & $\boldsymbol{W}$ & \multicolumn{4}{c|}{$\boldsymbol{J}_{\mathbf{H H}}(\mathbf{H z})$} \\
\hline & $(\mathrm{ppm})$ & $(\mathrm{Hz})$ & $\mathrm{H}^{\mathrm{d}}$ & $\mathrm{H}^{\mathrm{e}}$ & $\mathrm{H}^{\mathrm{a}}$ & $\mathrm{H}^{\mathrm{b}}$ \\
\hline $\mathbf{H}^{\mathrm{d}}$ & 4.282 & 1.20 & & & & \\
\hline $\mathbf{H}^{\mathrm{e}}$ & 4.170 & 1.20 & -9.00 & & & \\
\hline $\mathbf{H}^{\mathrm{a}}$ & 4.150 & 1.20 & 0.00 & 0.00 & & \\
\hline $\mathbf{H}^{\mathbf{b}}$ & 2.457 & 1.40 & 10.60 & 10.20 & 5.20 & \\
\hline $\mathbf{H}^{\mathbf{c}}$ & 2.133 & 1.20 & 3.20 & 9.30 & 0.00 & -13.00 \\
\hline
\end{tabular}
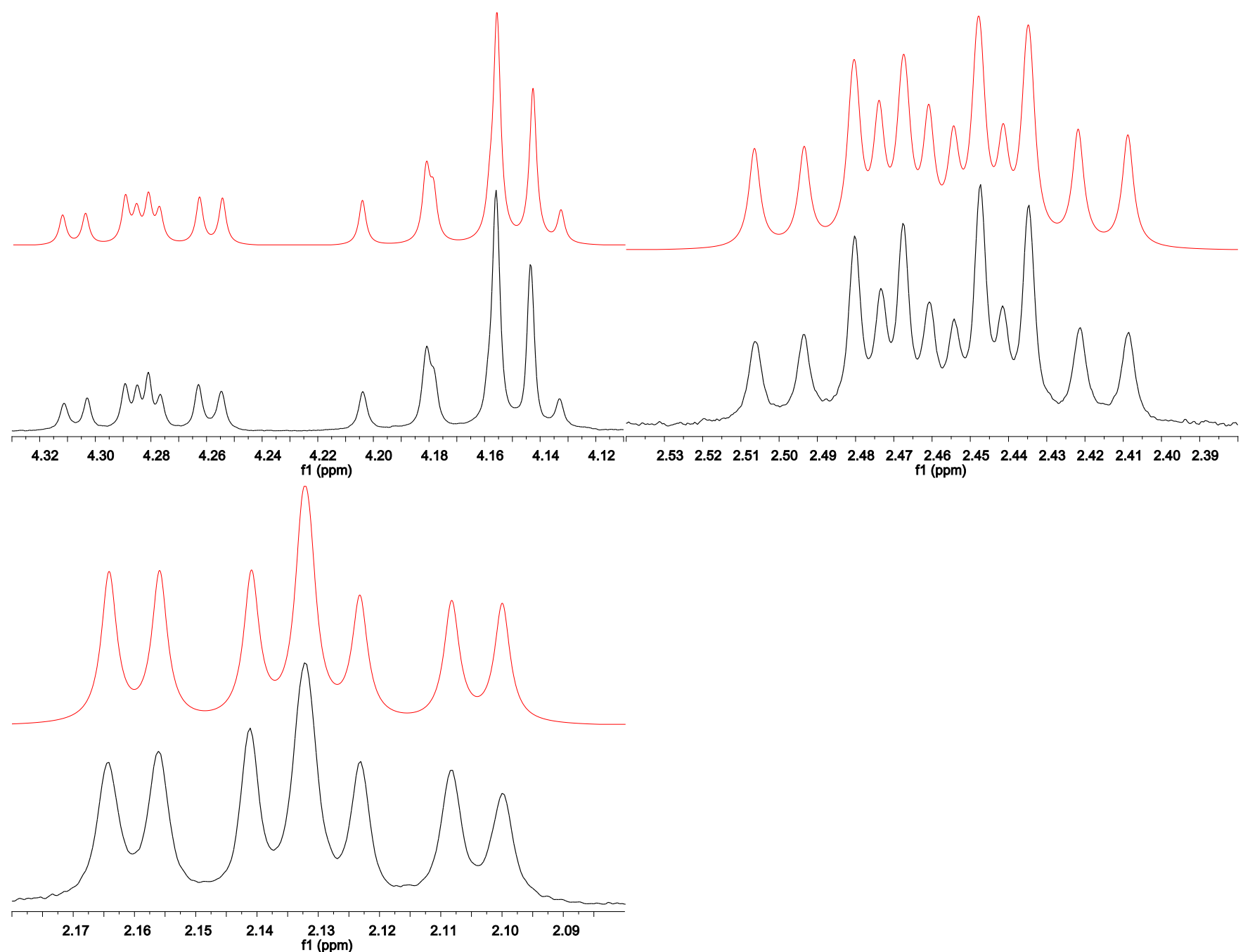

Figure S12. ${ }^{1} \mathrm{H}$ NMR sectra of $9 \mathrm{c}$ measured at $25^{\circ} \mathrm{C}$ (Black) and results of the simulations (red) showing the dihydrofuranyl moiety region $\left(400 \mathrm{MHz},\left[\mathrm{D}_{6}\right]\right.$ benzene). 


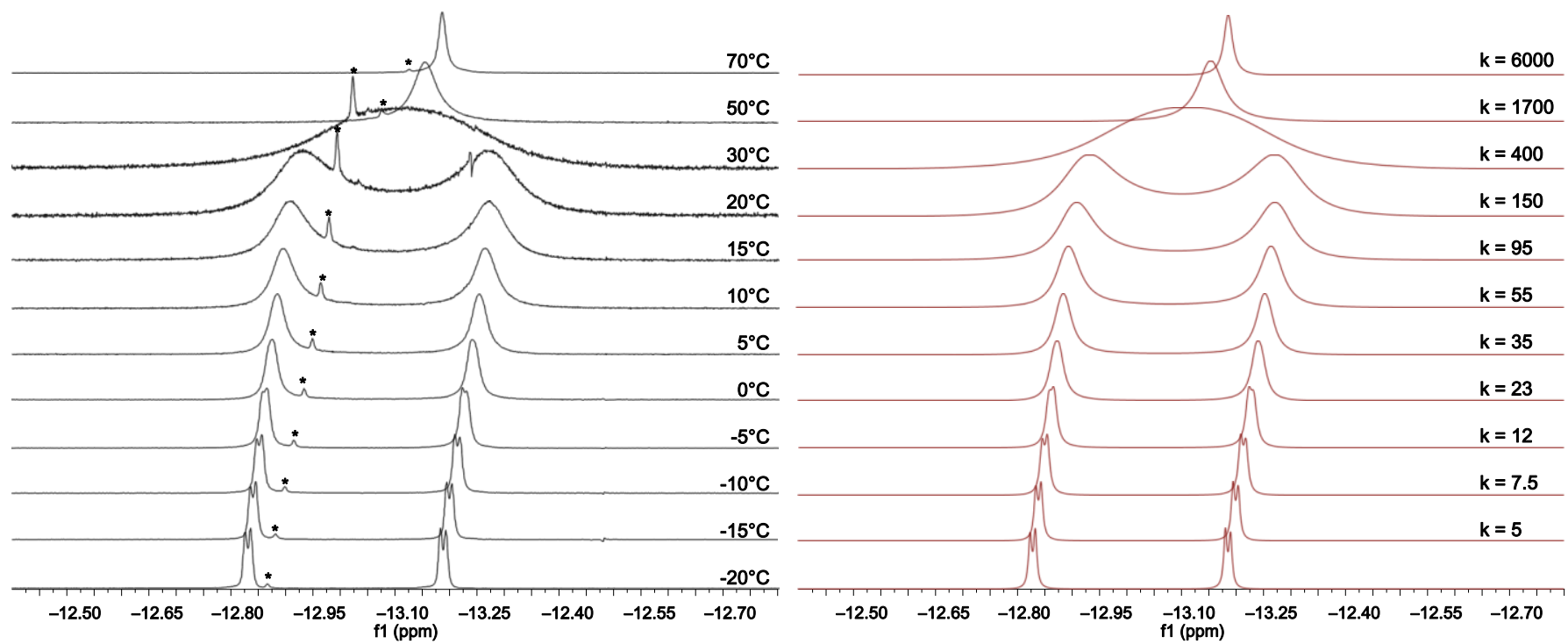

Figure S13. VT- ${ }^{1}$ H NMRof 5a (left) and simulated nmr spectrum (right). Hydride region. Impurity is denoted as an asterisk. (400 MHz, [D 8 toluene)

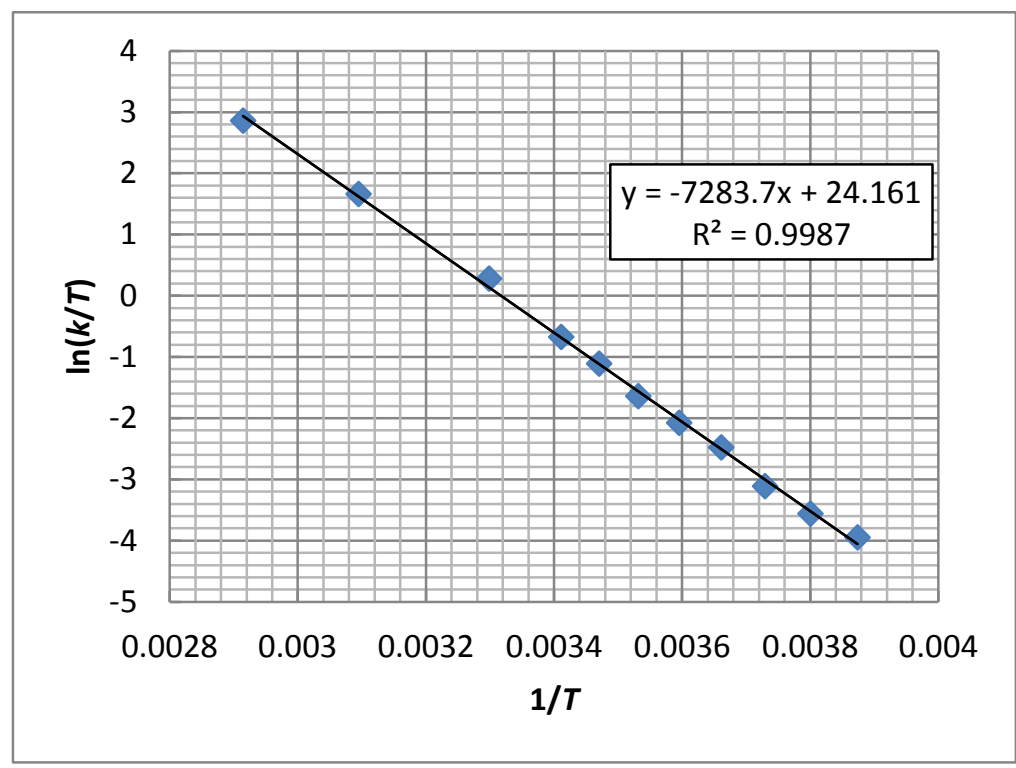

Figure S14. Eyring plot of 5a.

$\Delta H^{*}=60.6 \pm 1.7 \mathrm{~kJ} / \mathrm{mol}, \Delta S^{\ddagger}=3.3 \pm 5.8 \mathrm{~J} / \mathrm{mol} \mathrm{K}, \Delta G^{\ddagger}=52.3 \mathrm{~kJ} / \mathrm{mol}(298 \mathrm{~K})$ 
VT-NMR of $\mathbf{5 c}$.

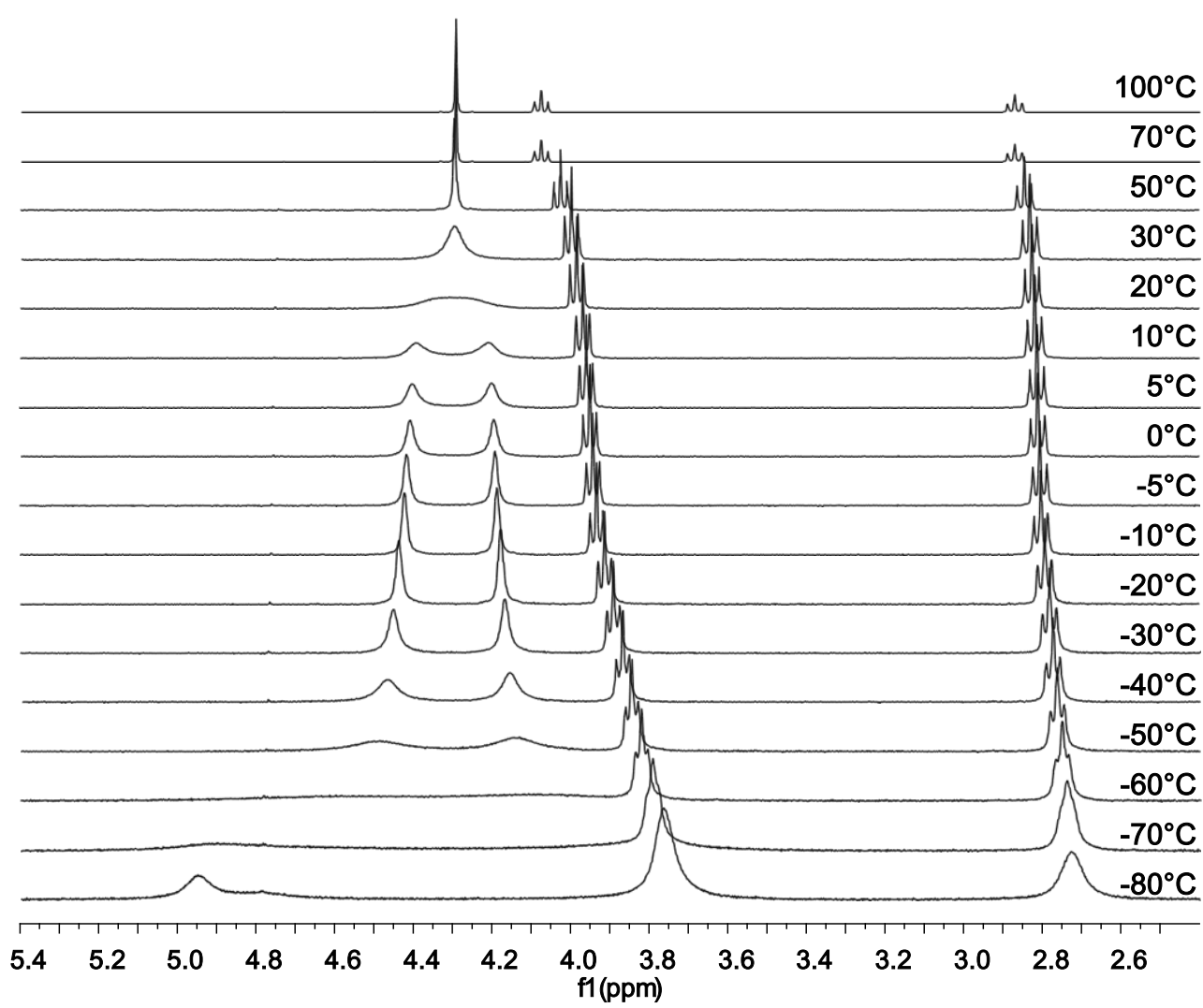

(a) Aromatic and oxycarbene region

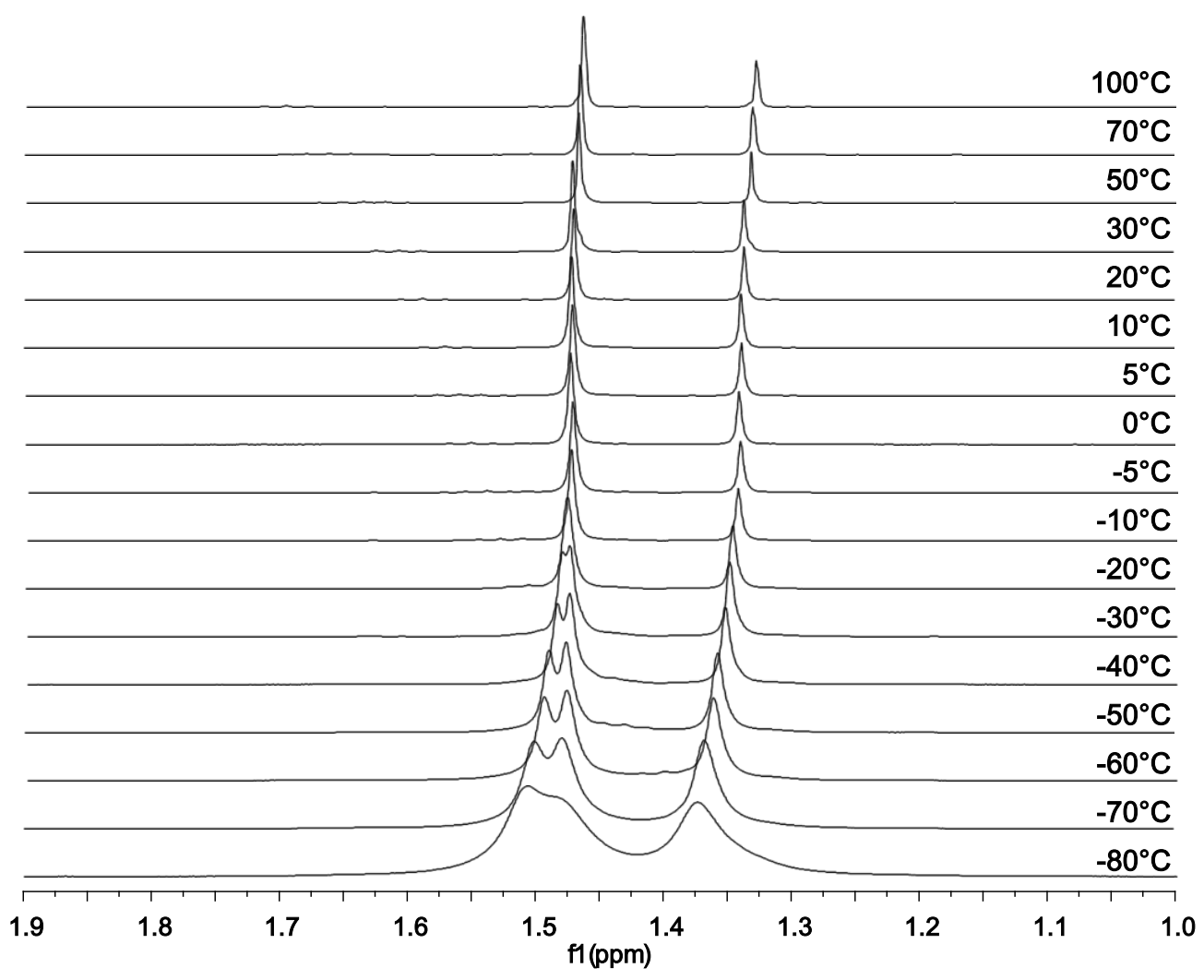

(b) $t$-Bu region 


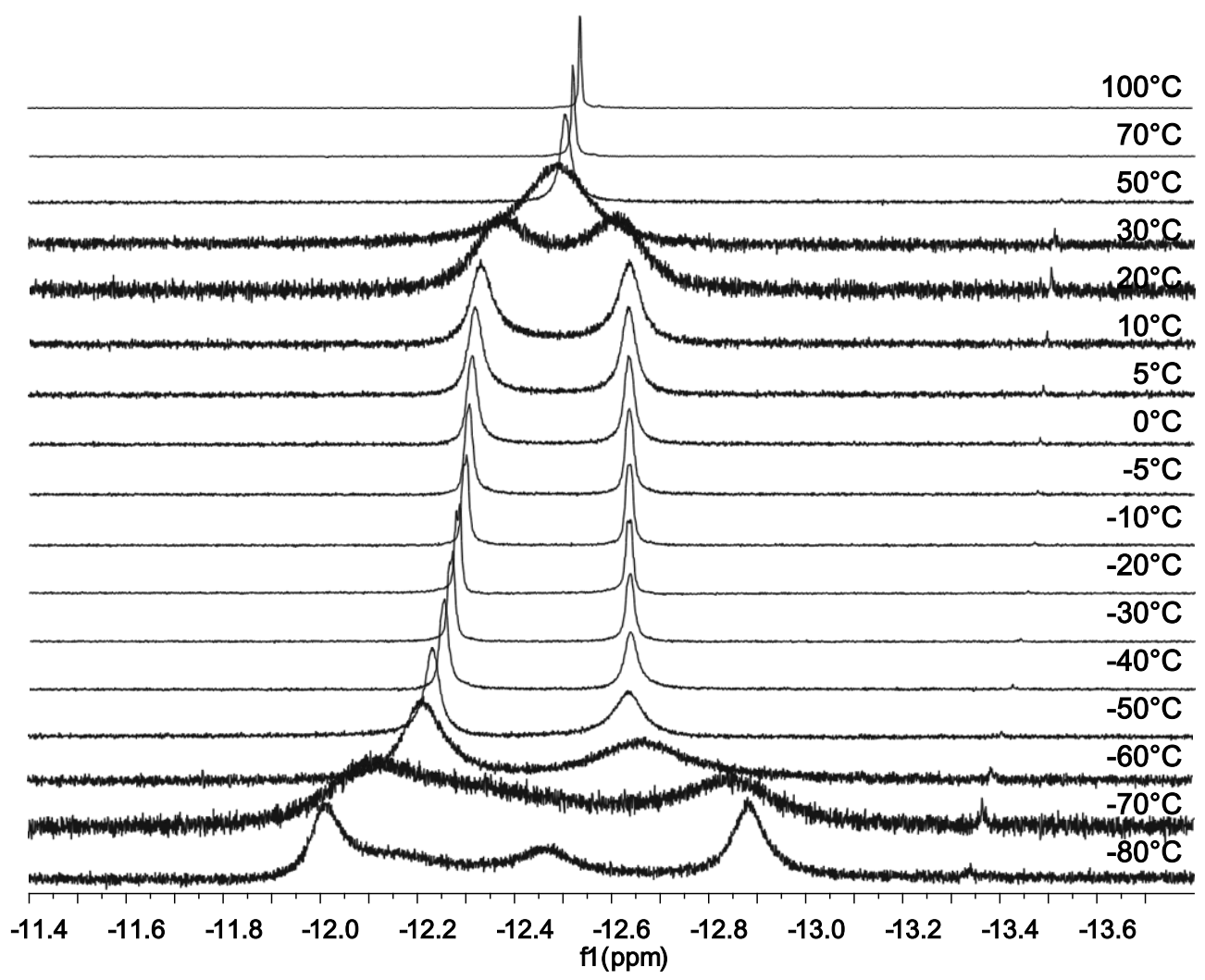

(c) Hydride region

Figure S15. VT- ${ }^{1} \mathrm{H}$ NMR spectrum of $\mathbf{5 c} .\left(400 \mathrm{MHz},\left[\mathrm{D}_{8}\right]\right.$ toluene)

The rate for exchange of the hydride signals at coalescence temperature $\left(30^{\circ} \mathrm{C}\right)$ was calculated as $k=313.5 \mathrm{~s}^{-1}$ using $\Delta v=$ $141.2 \mathrm{~Hz}$ at $-20^{\circ} \mathrm{C}$. The $\Delta G^{\ddagger}$ at $30^{\circ} \mathrm{C}$ was calculated as $\Delta G^{\ddagger}=59.8 \mathrm{~kJ} / \mathrm{mol}$.

The rate for exchange of the $\mathrm{CpH}$ signals at coalescence temperature $\left(20^{\circ} \mathrm{C}\right)$ was calculated as $k=230.9 \mathrm{~s}^{-1} \mathrm{using} \Delta v=103.9$ $\mathrm{Hz}$ at $-20^{\circ} \mathrm{C}$. The $\Delta G^{\ddagger}$ at $30^{\circ} \mathrm{C}$ was calculated as $\Delta G^{\ddagger}=58.5 \mathrm{~s} \mathrm{~kJ} / \mathrm{mol}$. 
5 NMR spectrum of $\mathbf{5 a}, \mathbf{5 b}, \mathbf{5 c}$, cis-8a, trans-8a, 9a, 9c, and $\mathbf{1 0 a}$.

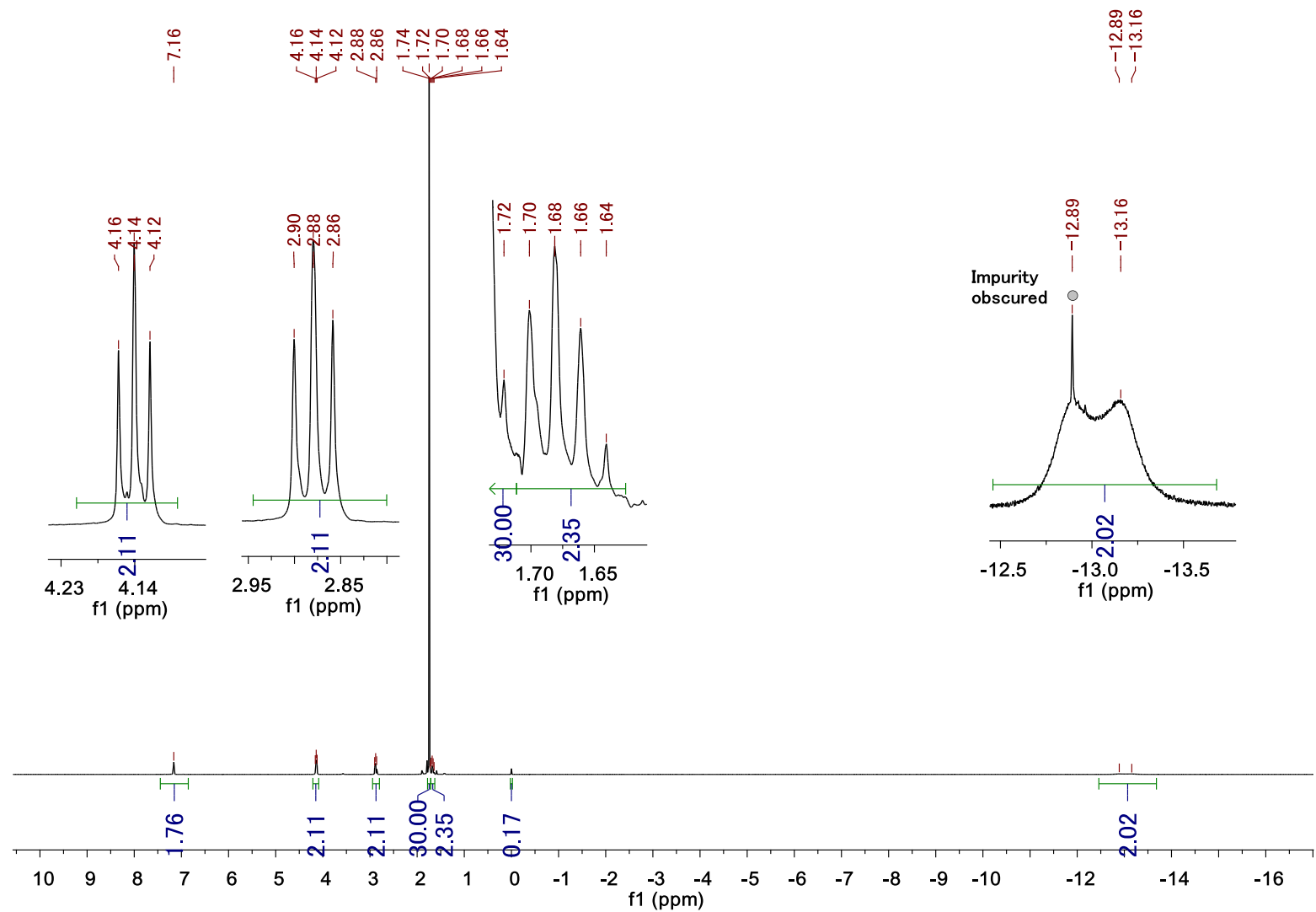

Figure S16. ${ }^{1} \mathrm{H}$ NMR (400 MHz, $25{ }^{\circ} \mathrm{C}$, [D 6 ]benzene) of $\mathbf{5 a}$.

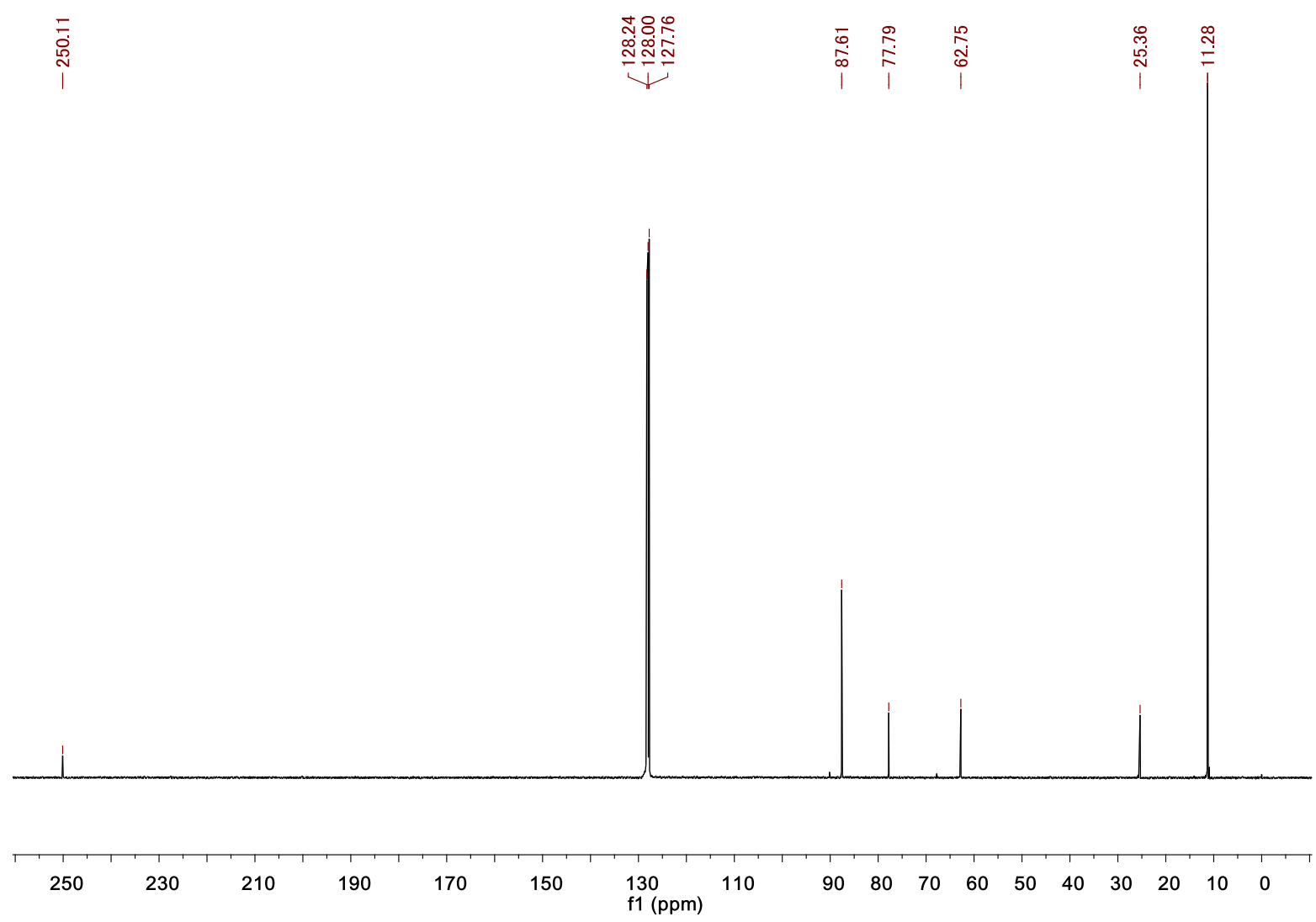

Figure S17. ${ }^{13} \mathrm{C}\left\{{ }^{1} \mathrm{H}\right\}$ NMR $\left(100 \mathrm{MHz}, 25{ }^{\circ} \mathrm{C},\left[\mathrm{D}_{6}\right]\right.$ benzene $)$ of 5a. 


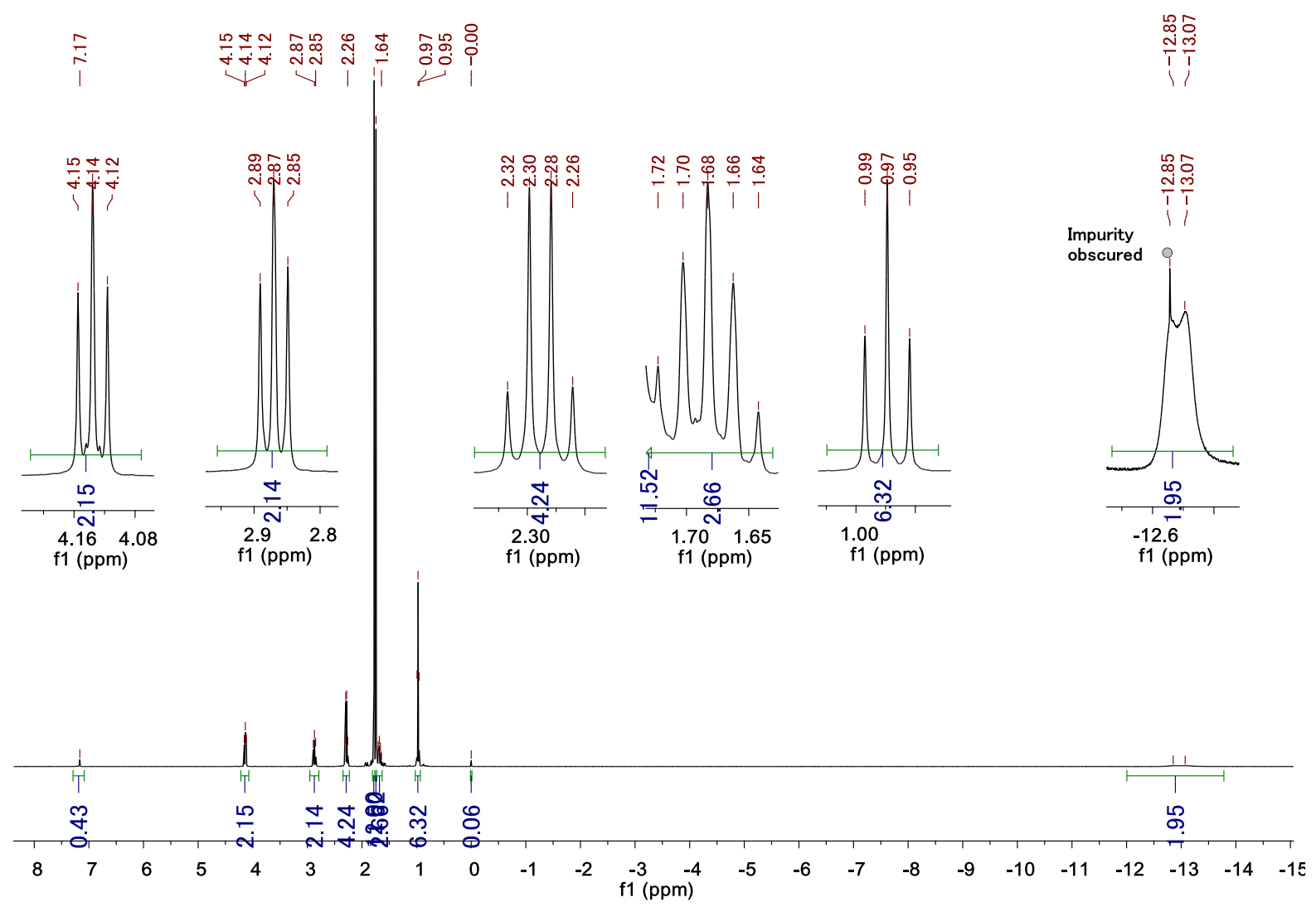

Figure S18. ${ }^{1} \mathrm{H}$ NMR $\left(400 \mathrm{MHz}, 25{ }^{\circ} \mathrm{C},\left[\mathrm{D}_{6}\right]\right.$ benzene) of $\mathbf{5 b}$.

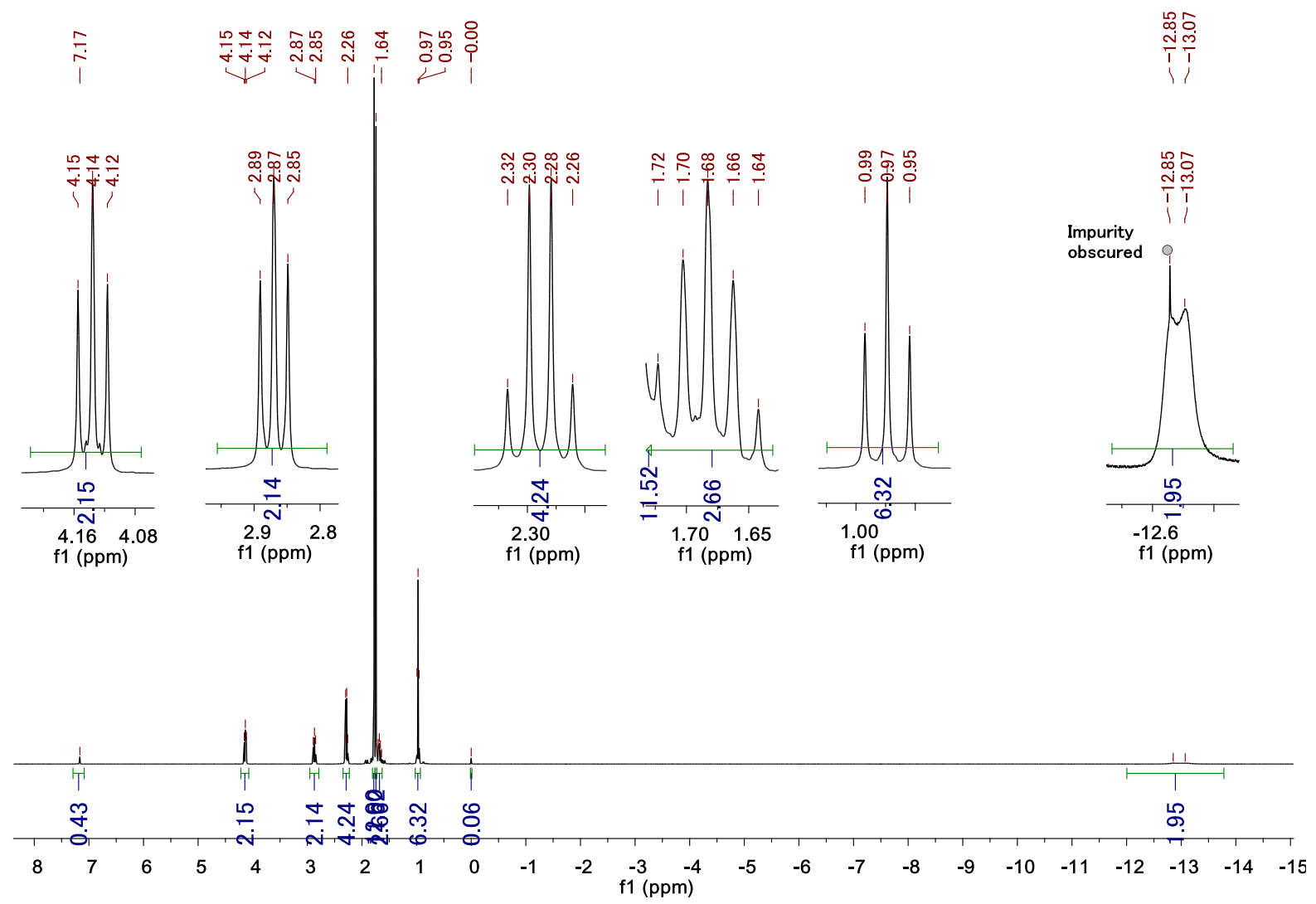

Figure $\mathrm{S} 19 .{ }^{13} \mathrm{C}\left\{{ }^{1} \mathrm{H}\right\}$ NMR $\left(100 \mathrm{MHz}, 25{ }^{\circ} \mathrm{C},\left[\mathrm{D}_{6}\right]\right.$ benzene) of $\mathbf{5 b}$. 


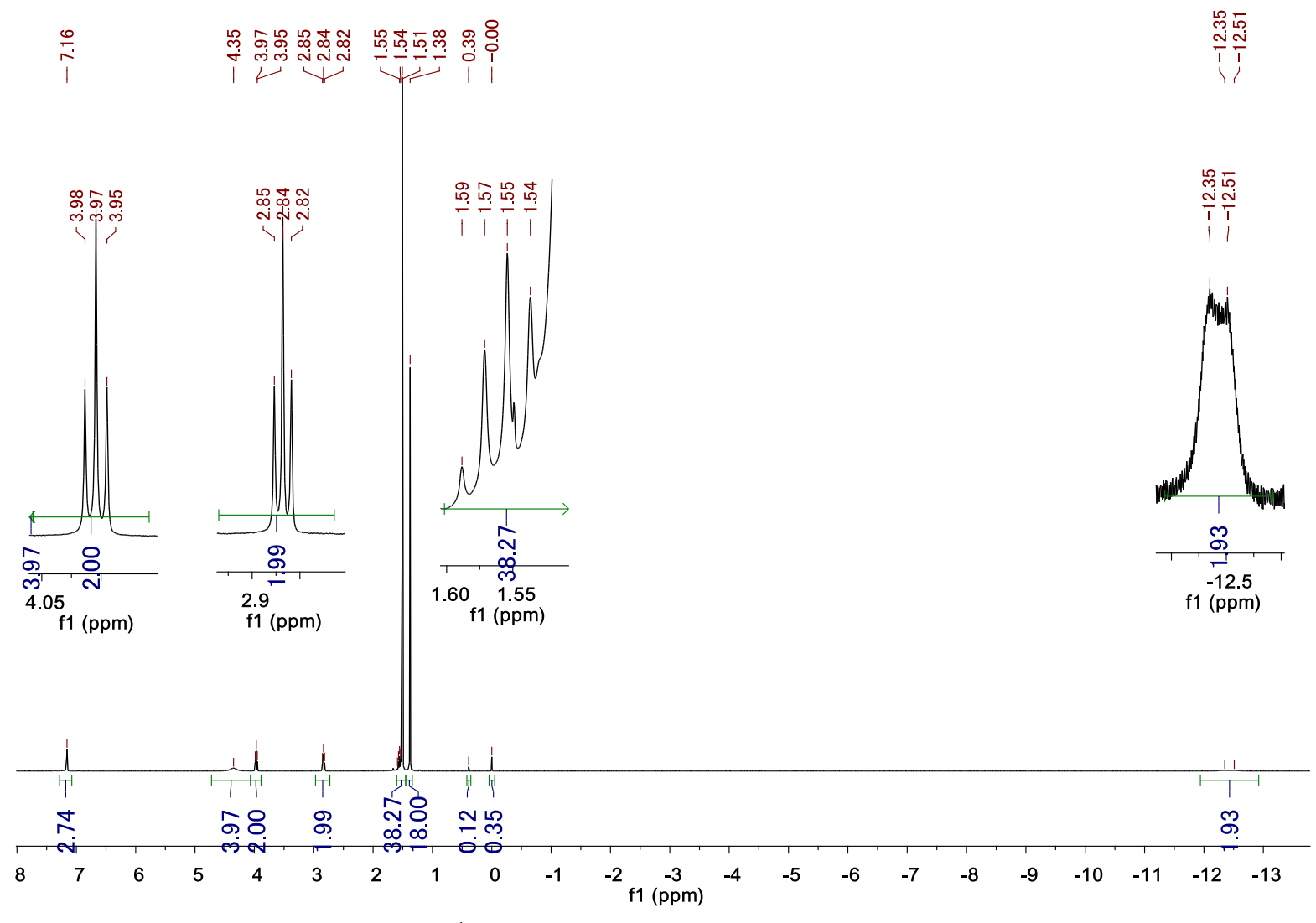

Figure S20. ${ }^{1} \mathrm{H}$ NMR (400 MHz, $25{ }^{\circ} \mathrm{C},\left[\mathrm{D}_{6}\right]$ benzene) of $\mathbf{5 c}$.

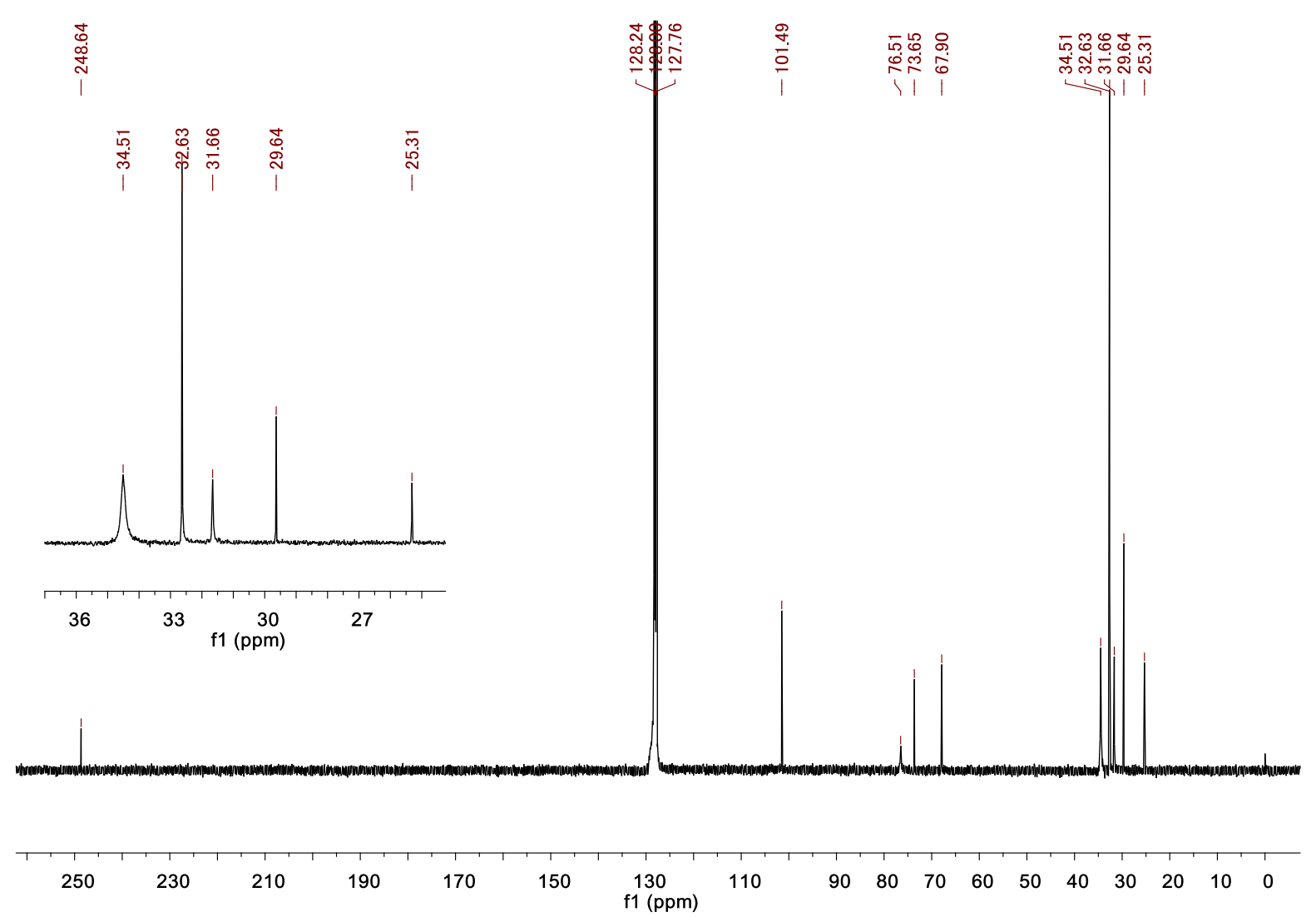

Figure $\mathrm{S} 21 .{ }^{13} \mathrm{C}\left\{{ }^{1} \mathrm{H}\right\}$ NMR $\left(100 \mathrm{MHz}, 25{ }^{\circ} \mathrm{C},\left[\mathrm{D}_{6}\right]\right.$ benzene $)$ of $\mathbf{5 c}$. 


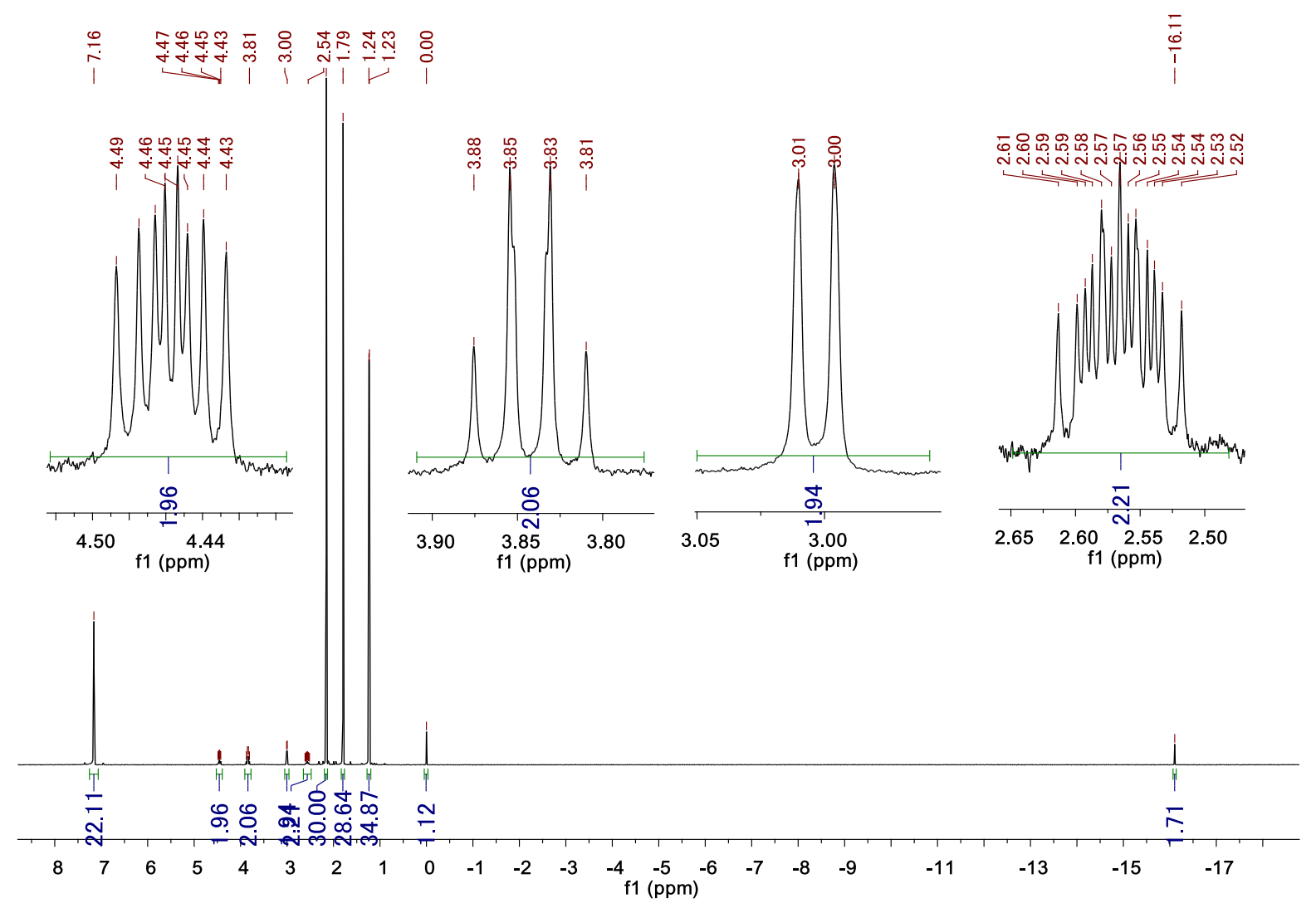

Figure S22. ${ }^{1} \mathrm{H}$ NMR $\left(400 \mathrm{MHz}, 25^{\circ} \mathrm{C}\right.$, [D $\left.\mathrm{D}_{6}\right]$ benzene) of cis-8a.

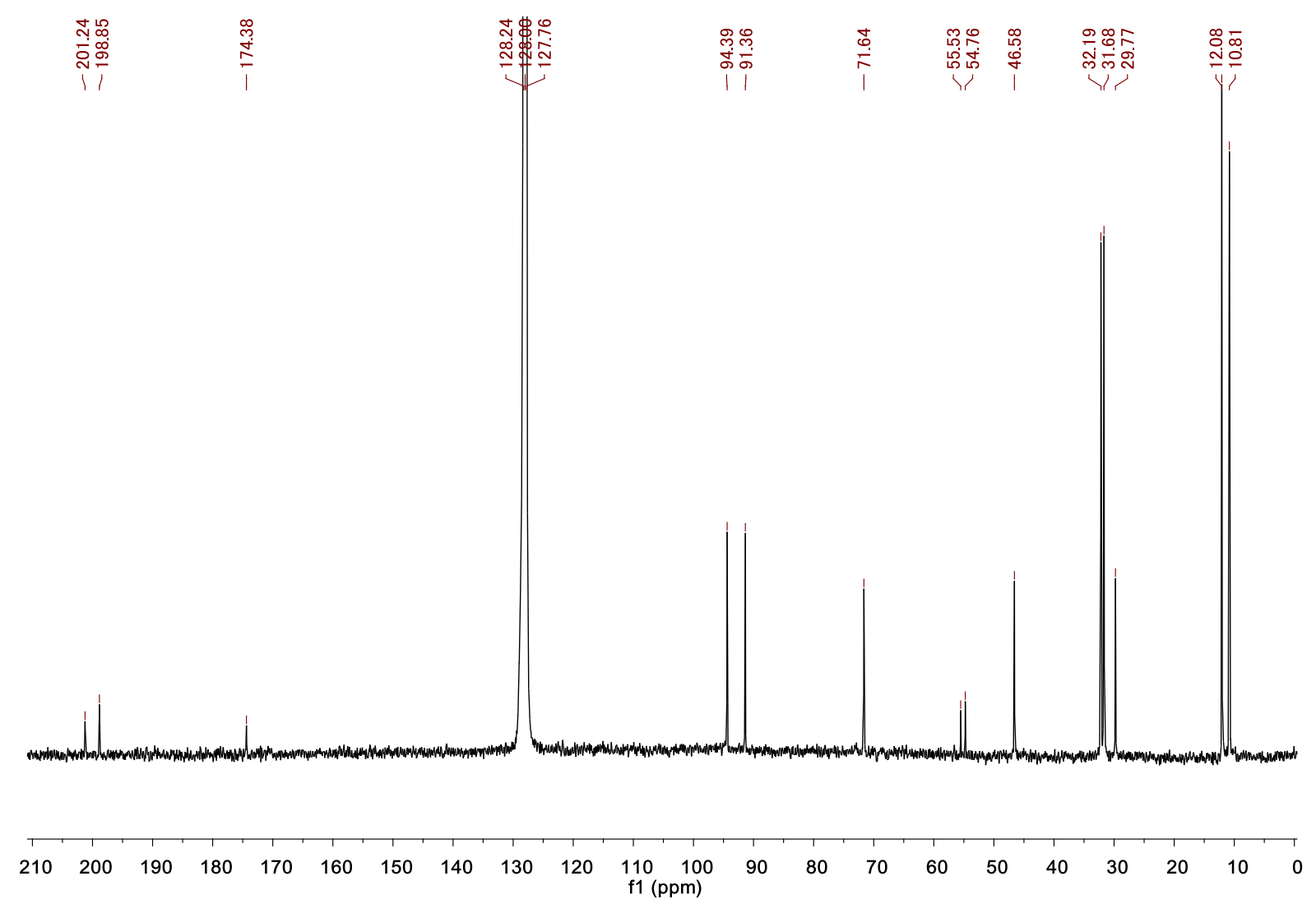

Figure S23. ${ }^{13} \mathrm{C}\left\{{ }^{1} \mathrm{H}\right\}$ NMR $\left(100 \mathrm{MHz}, 25{ }^{\circ} \mathrm{C},\left[\mathrm{D}_{6}\right]\right.$ benzene $)$ of cis-8a. 


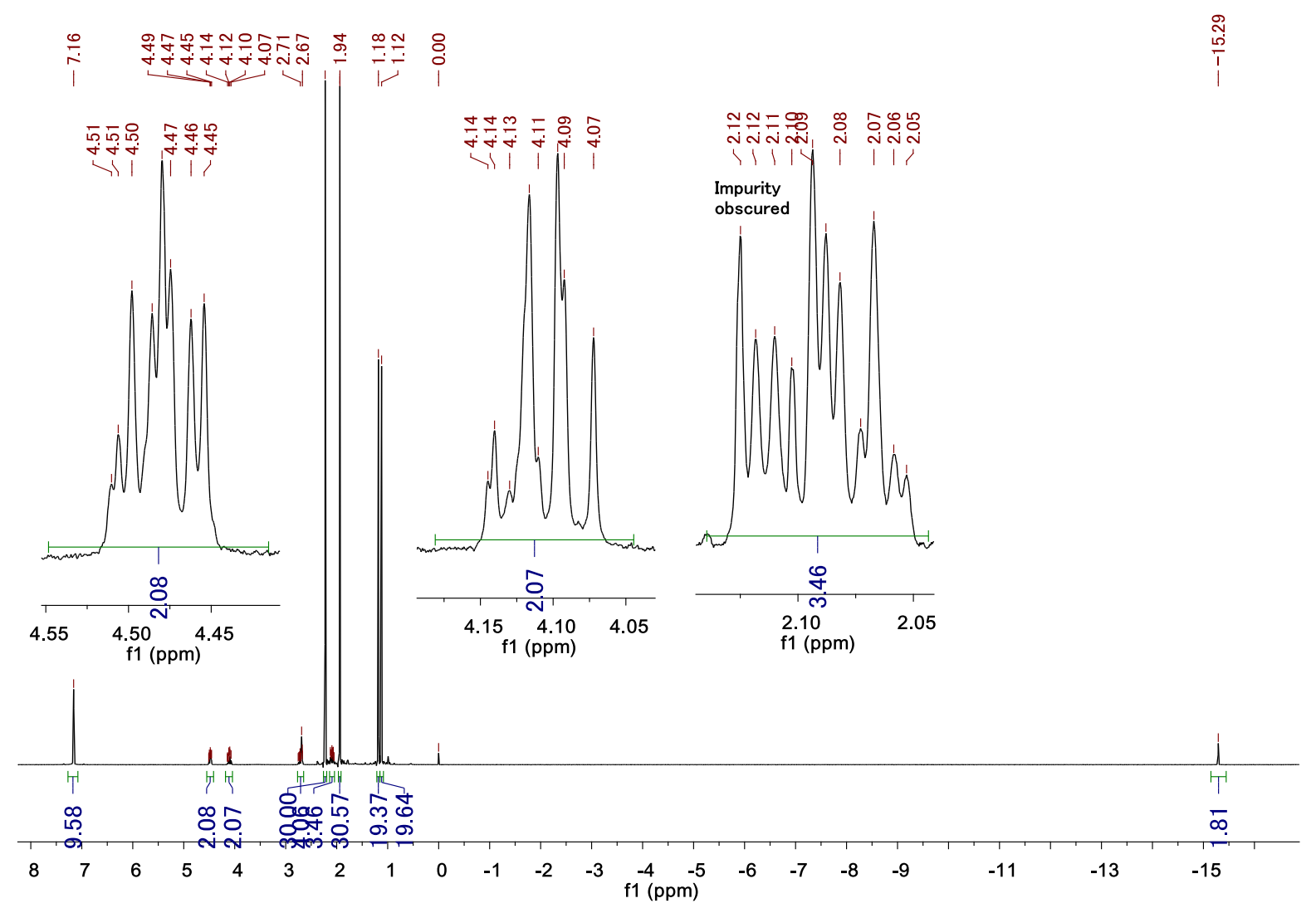

Figure S24. ${ }^{1} \mathrm{H}$ NMR $\left(400 \mathrm{MHz}, 25{ }^{\circ} \mathrm{C},\left[\mathrm{D}_{6}\right]\right.$ benzene) of trans-8a.

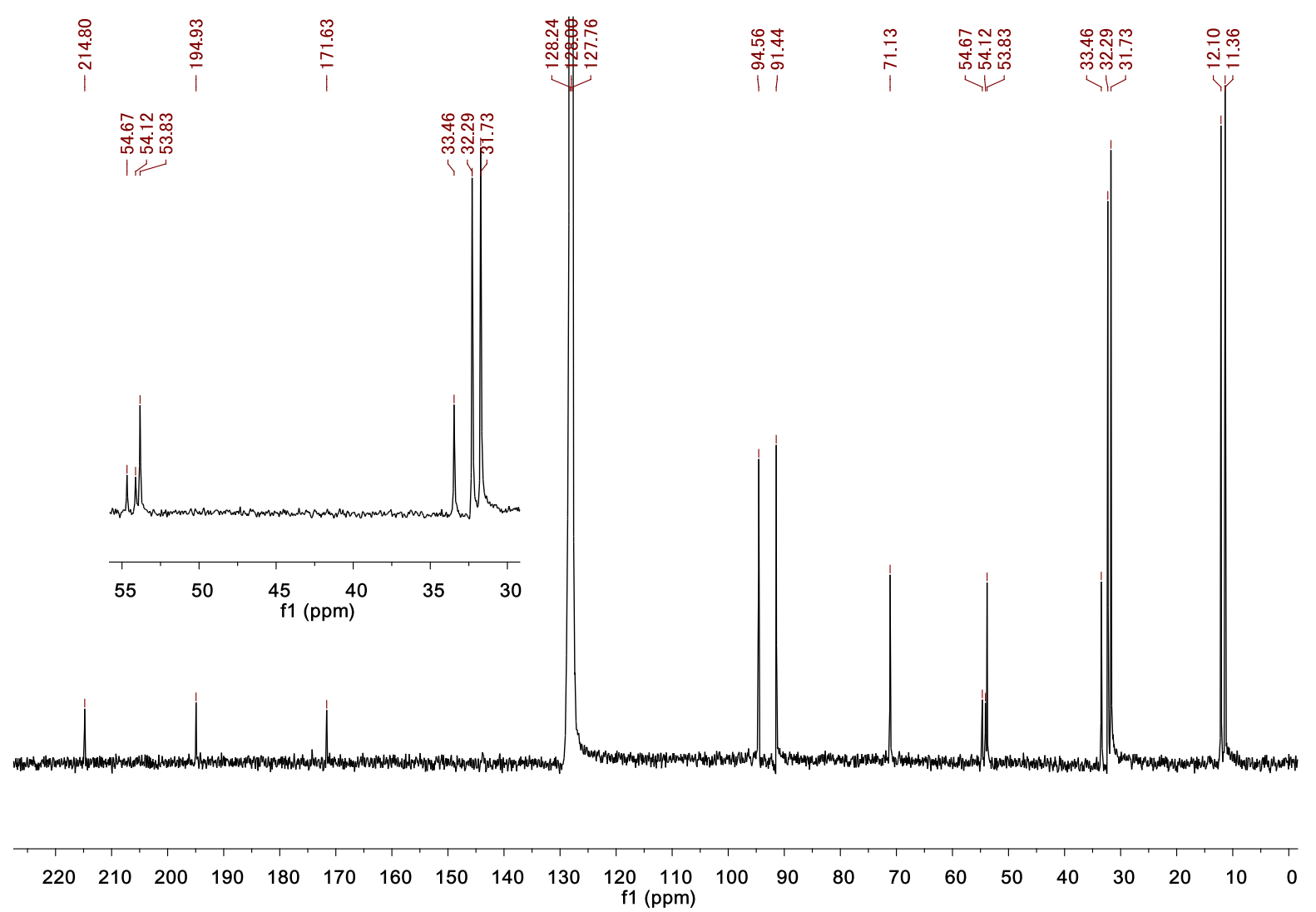

Figure S25. ${ }^{13} \mathrm{C}\left\{{ }^{1} \mathrm{H}\right\}$ NMR $\left(100 \mathrm{MHz}, 25{ }^{\circ} \mathrm{C},\left[\mathrm{D}_{6}\right]\right.$ benzene $)$ of trans-8a. 


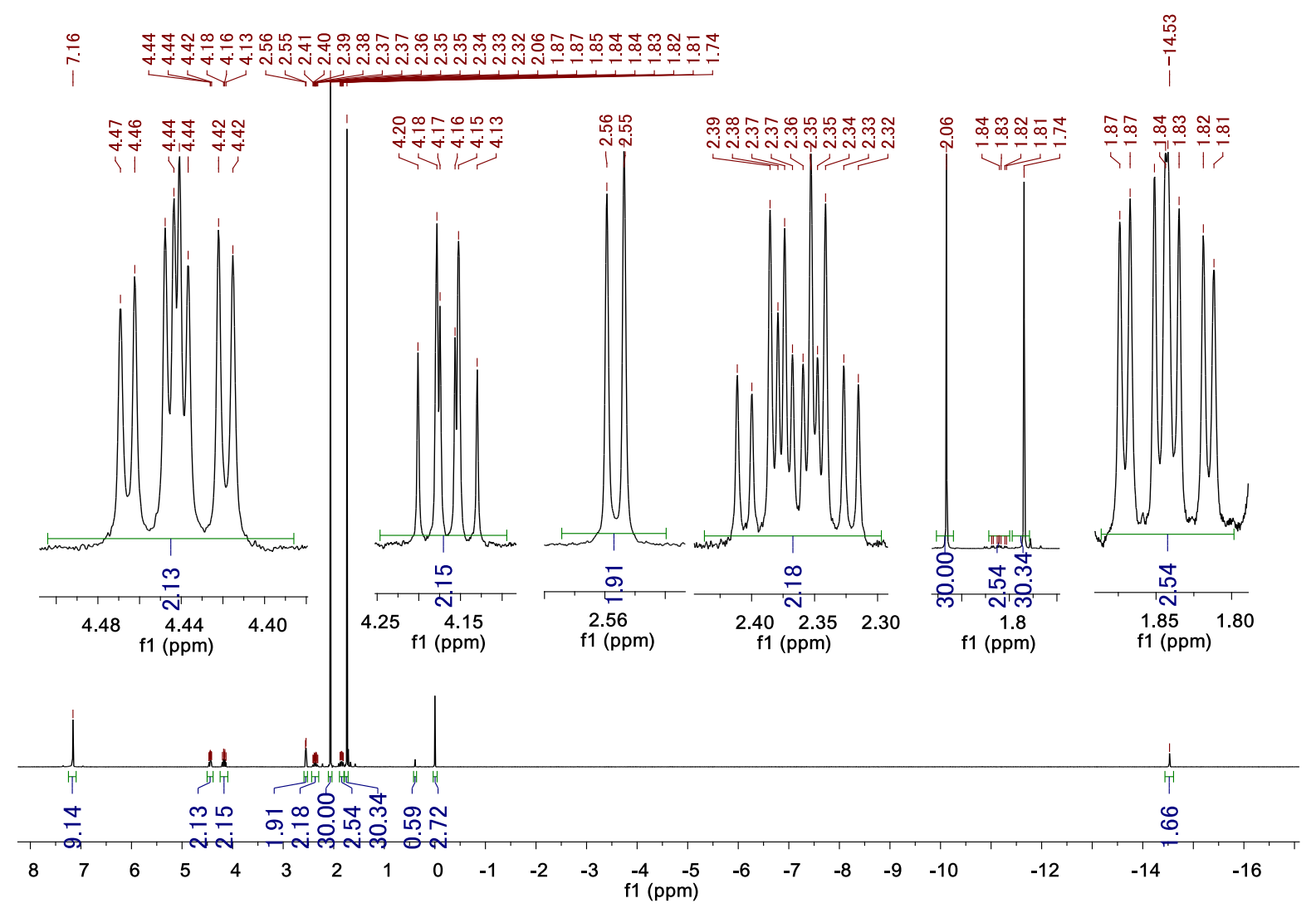

Figure S26. ${ }^{1} \mathrm{H}$ NMR $\left(400 \mathrm{MHz}, 25{ }^{\circ} \mathrm{C},\left[\mathrm{D}_{6}\right]\right.$ benzene) of $9 \mathbf{a}$.

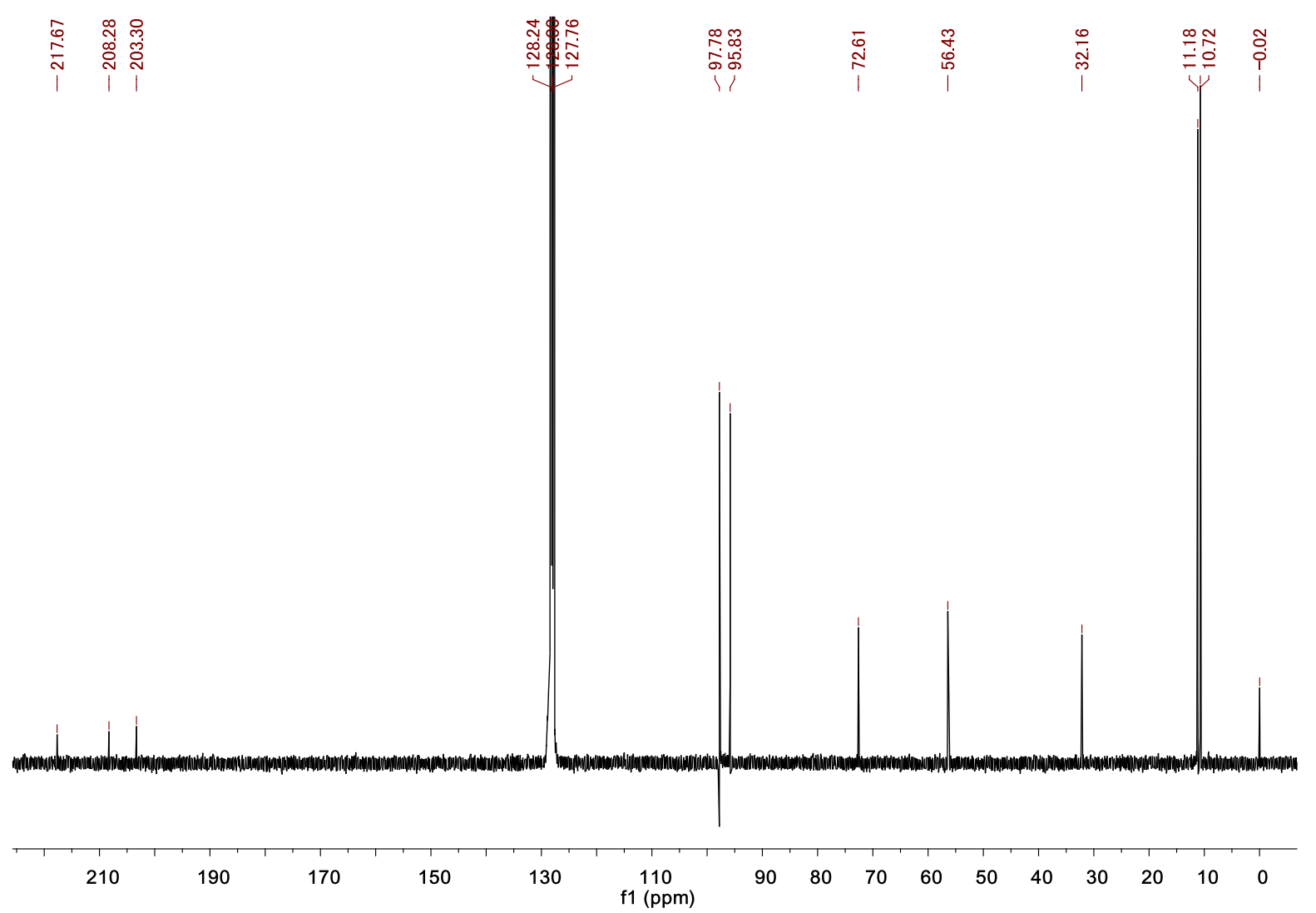

Figure S27. ${ }^{13} \mathrm{C}\left\{{ }^{1} \mathrm{H}\right\}$ NMR $\left(100 \mathrm{MHz}, 25{ }^{\circ} \mathrm{C},\left[\mathrm{D}_{6}\right]\right.$ benzene $)$ of $9 \mathbf{a}$. 


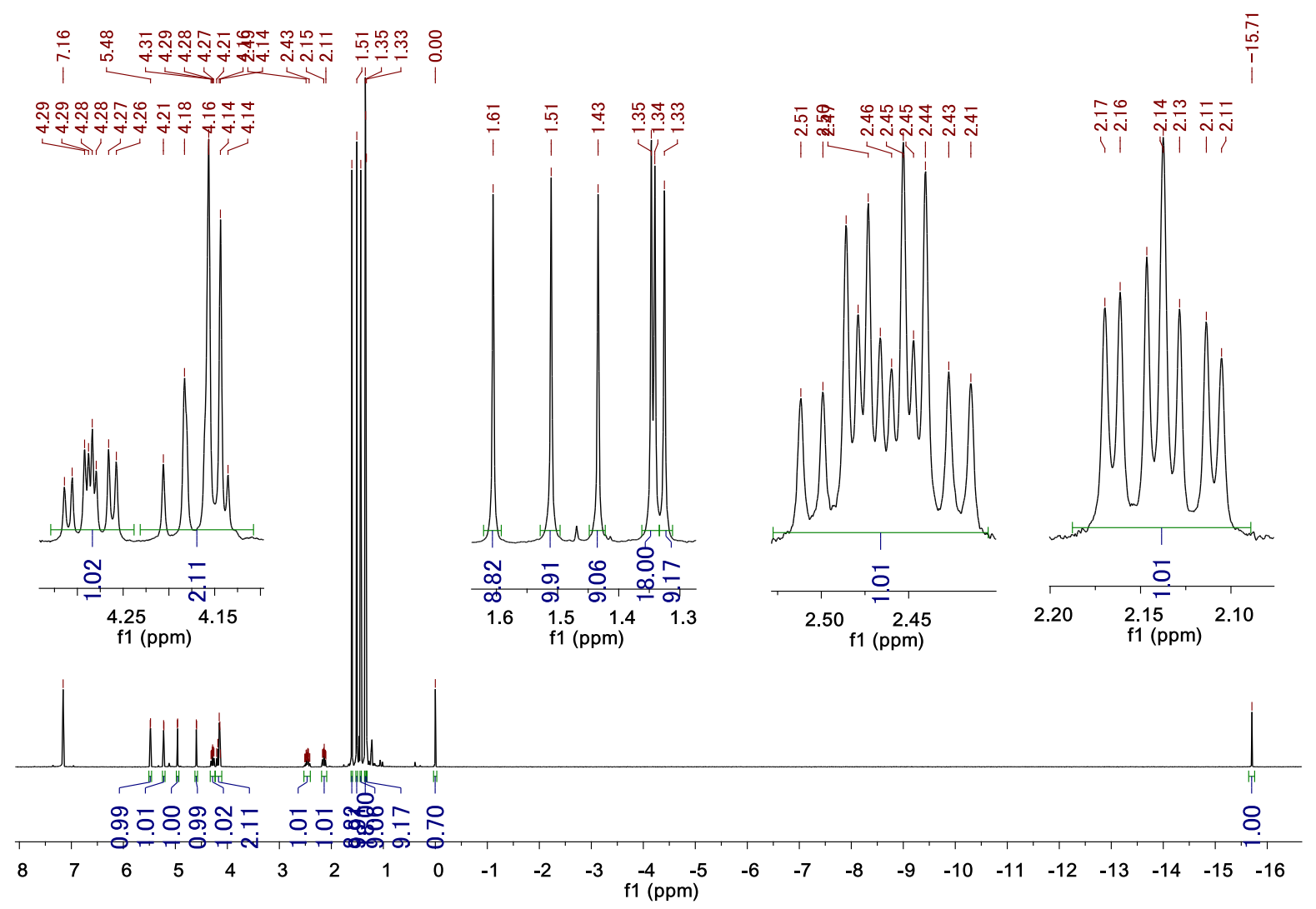

Figure S28. ${ }^{1} \mathrm{H}$ NMR (400 MHz, $25{ }^{\circ} \mathrm{C},\left[\mathrm{D}_{6}\right]$ benzene) of $\mathbf{9 c}$.

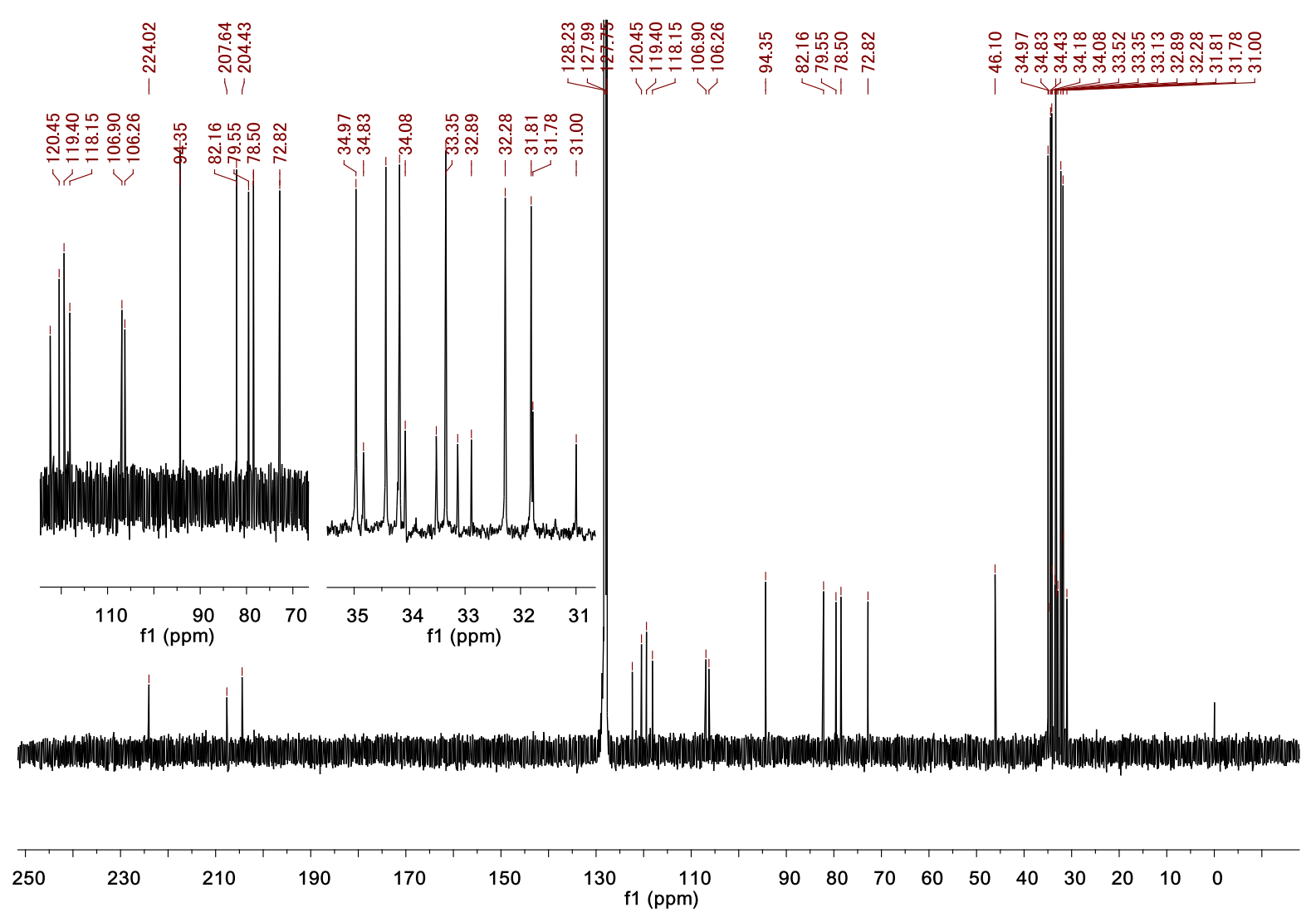

Figure S29. ${ }^{13} \mathrm{C}\left\{{ }^{1} \mathrm{H}\right\}$ NMR $\left(100 \mathrm{MHz}, 25{ }^{\circ} \mathrm{C},\left[\mathrm{D}_{6}\right]\right.$ benzene $)$ of $9 c$. 


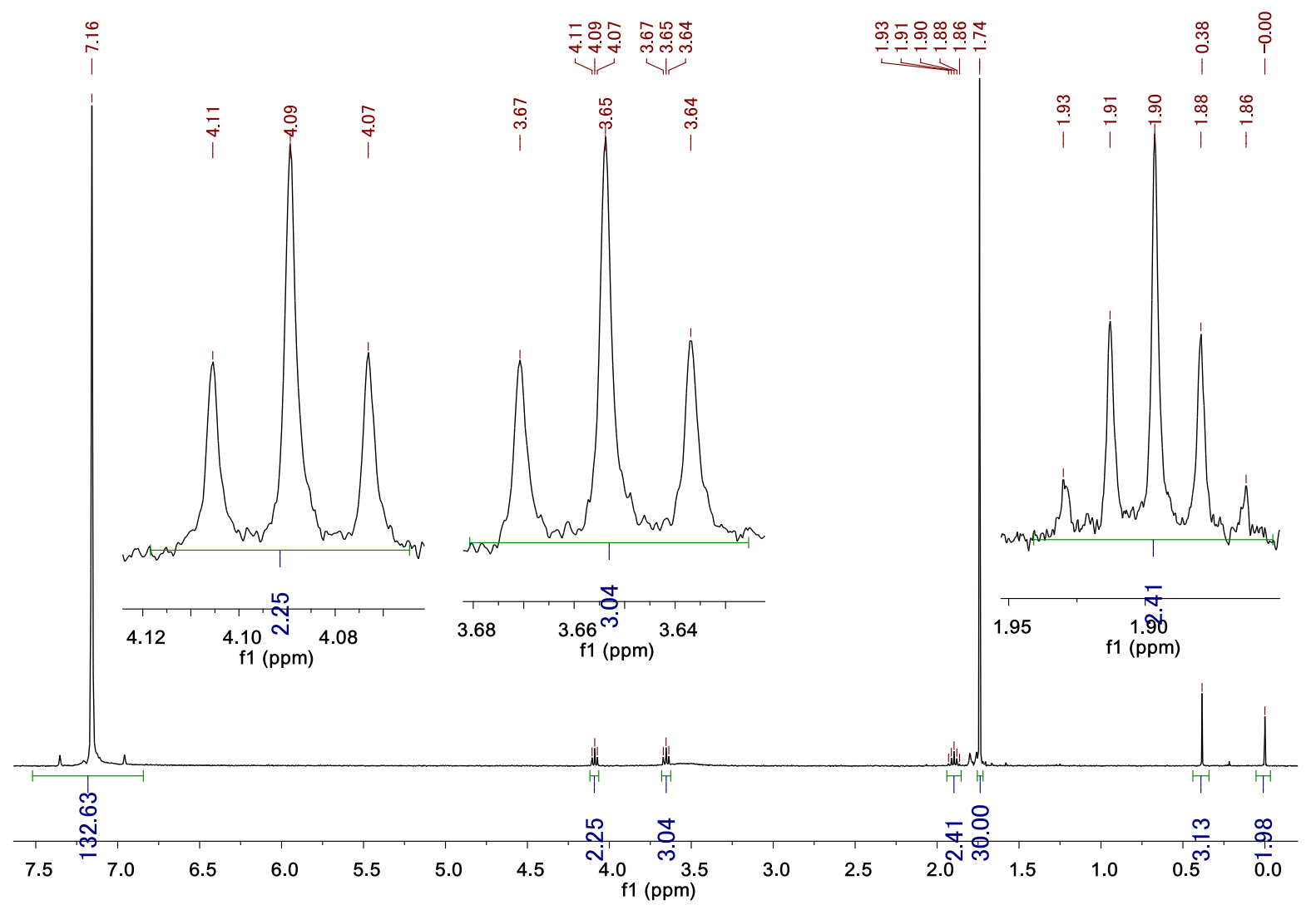

Figure S30. ${ }^{1} \mathrm{H}$ NMR $\left(400 \mathrm{MHz}, 25^{\circ} \mathrm{C},\left[\mathrm{D}_{6}\right]\right.$ benzene) of $\mathbf{1 0 a}$. 